Published in final edited form as:

J Med Chem. 2016 June 9; 59(11): 5377-5390. doi:10.1021/acs.jmedchem.6b00236.

\title{
Thiolactomycin-based inhibitors of bacterial $\beta$-ketoacyl-ACP synthases with in vivo activity
}

\author{
Gopal R. Bommineni ${ }^{\dagger, \ddagger}$, Kanishk Kapilashrami ${ }^{\dagger, \ddagger, \AA}$, Jason E. Cummings ${ }^{\S}$, Yang Lu $^{\dagger}$, Susan \\ E. Knudson ${ }^{\S}$, Chendi Gu ${ }^{\dagger}$, Stephen G. Walker ${ }^{¥}$, Richard A. Slayden ${ }^{\S}{ }^{*}$, and Peter J. Tonge ${ }^{\dagger,{ }^{*}}$ \\ †Institute of Chemical Biology \& Drug Discovery, Department of Chemistry \\ ${ }^{\sharp}$ School of Dental Medicine, Department of Oral Biology and Pathology, Stony Brook University, \\ Stony Brook, NY 11794-3400 \\ $\S$ Department of Microbiology, Immunology and Pathology, Colorado State University, Fort Collins, \\ CO 80523-2025
}

\begin{abstract}
$\beta$-Ketoacyl-ACP synthases (KAS) are key enzymes involved in the type II bacterial fatty acid biosynthesis (FASII) pathway and are putative targets for antibacterial discovery. Several natural product KAS inhibitors have previously been reported, including thiolactomycin (TLM), which is produced by Nocardia spp. Here we describe the synthesis and characterization of optically pure $5 R$-thiolactomycin (TLM) analogs that show improved whole cell activity against bacterial strains including methicillin resistant Staphylococcus aureus (MRSA) and priority pathogens such as Francisella tularensis and Burkholderia pseudomallei. In addition, we identify TLM analogs with in vivo efficacy against MRSA and Klebsiella pneumoniae in animal models of infection.
\end{abstract}

\section{Graphical Abstract}

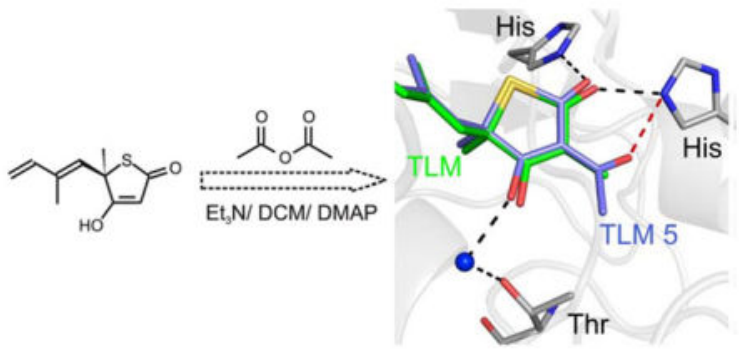

\footnotetext{
"Corresponding Author: Authors for correspondence: RAS. Tel: (970) 491 2902, Fax: (970) 491 1815, richard.slayden@ colostate.edu. PJT. Tel: (631) 632 7907, Fax: (631) 632 7960, peter.tonge@ stonybrook.edu. \$GRB and KK contributed equally.

IlCurrent Address: Molecular Pharmacology and Chemistry Program, Zuckerman Research Center, Memorial Sloan-Kettering Cancer Center, 417 East 68th Street, New York, NY 10065

Author Contributions

The manuscript was written through contributions of all authors. All authors have given approval to the final version of the manuscript. Supporting Information. ${ }^{13} \mathrm{C},{ }^{1} \mathrm{H}$ NMR spectra, primers used for cloning saFabF, detailed material and methods for assessing the whole cell activity of the TLM analogs. This material is available free of charge via the internet at http://pubs.acs.org.
} 


\section{Keywords}

Thiolactomycin; Fatty Acid Biosynthesis; $\beta$-Ketoacyl ACP synthase; Structure Activity

Relationships; in vitro activity; in vivo efficacy

\section{INTRODUCTION}

While antibiotic resistance has always been a driving force for drug discovery, the increase in the number and the diversity of resistant pathogens continues to pose a severe threat to public health. ${ }^{1}$ Multidrug resistant strains of organisms such as Klebsiella pneumoniae and Acinetobacter baumannii are virtually untreatable by currently available antibiotics. ${ }^{1-3}$ Moreover, resistance has been reported towards drugs such as vancomycin, that is considered the 'drug of last resort' for a variety of bacterial infections including Staphylococcus aureus. ${ }^{4}$ Thus there is a critical need for antibacterial agents with novel modes of action that circumvent existing resistance mechanisms.

The bacterial type II fatty acid biosynthesis pathway (FASII, Figure 1) is highly conserved, essential for survival and is distinct from the mammalian pathway. ${ }^{5-7}$ Thus, enzymes in this pathway are thought to be profitable targets for the development of novel therapeutics. In support of this proposal, the front-line tuberculosis drug isoniazid is an inhibitor of the enoyl-ACP reductase (FabI) in the Mycobacterium tuberculosis FASII pathway, ${ }^{8-11}$ while several FabI inhibitors are currently in clinical or preclinical development to treat infections caused by methicillin-resistant $S$. aureus (MRSA). ${ }^{12-14}$

In addition to FabI, the FASII $\beta$-ketoacyl-ACP synthase (KAS) enzymes are also thought to be appropriate targets for intervention. These enzymes catalyze a decarboxylative Claisen condensation in the initiation and elongation phases of the FASII pathway, and typically bacteria have three KAS homologs: FabB, FabF and FabH (Table 1). FabB and FabF (KASI/II) contain a Cys-His-His catalytic triad and catalyze the condensation of malonylACP and acyl-ACP in the elongation cycle, while FabH (KASIII) contains a Cys-His-Asn triad, and is responsible for initiation of the FASII cycle through the condensation of malonyl-ACP with acetyl-CoA. ${ }^{7},{ }^{15-18}$ More recently, Yuan et al. identified a new class of KASI/II enzymes (FabY) which replaces FabH in Pseudomonas aeruginosa. ${ }^{19}$

Although target redundancy adds an additional level of complexity for drug development, the identification of natural product KAS inhibitors lends support to the notion that this enzyme class is a valid target for drug discovery. ${ }^{7,20-23}$ Cerulenin, from the fungus Caephalosporium caerulens, is a suicide inhibitor that targets FabB and FabF in E. coli, ${ }^{7}$ whilst platensimycin, from Streptomyces platensis, ${ }^{23}$ is effective against Gram-positive bacteria such as $S$. aureus, Enterococcus faecalis and Streptococcus pneumonia. ${ }^{20} \mathrm{In}$ addition, the platensimycin analog platencin, inhibits both FabF and FabH comparably, and also is effective against various antibiotic resistant bacteria including vancomycin-resistant Enterococcus faecium. ${ }^{24}$ Finally phomallenic acids, isolated from a plant fungus, are KAS inhibitors and have potent antibacterial activity against MRSA, Bacillus subtilis and Haemophilus influenza. ${ }^{21,22}$ 
Thiolactomycin (TLM), a natural product thiolactone, is a competitive reversible inhibitor that binds to the malonyl-ACP binding site of the KAS enzymes. ${ }^{15}$ First isolated from Nocardia sp., ${ }^{25-27}$ TLM shows broad spectrum antibacterial activity against Gram-positive and Gram-negative bacteria, as well as mycobacteria. ${ }^{25,27,28}$ Despite moderate MIC values, the favorable physicochemical properties, low cytotoxicity, high bioavailability and in vivo activity of TLM, ${ }^{25-27,29-33}$ have stimulated a number of inhibitor design initiatives aimed at improving the antibacterial activity of this natural product. These synthetic efforts have mainly explored substitutions at the thiolactone $\mathrm{C} 5, \mathrm{O} 4$ and $\mathrm{C} 3$ positions, but in many cases have failed to improve the activity of TLM..$^{31,34-44}$ In addition, there are few reports of enantiomerically pure TLM analogs, ${ }^{45-51}$ and most studies have been performed with racemic mixtures of the respective TLM analogs despite the knowledge that (5R)-TLM is the biologically active enantiomer. ${ }^{43}$

Previously, we demonstrated that TLM is a time-dependent inhibitor of the FabB and FabF acyl-enzymes from $M$. tuberculosis and E. coli. ${ }^{32}$ Subsequently, interligand NOE NMR studies suggested that substituents at the $\mathrm{C} 4$ and the $\mathrm{C} 3$ positions of TLM (Figure 1) would strengthen interactions with the target enzyme, and subsequently it was demonstrated that modifications to these positions on the thiolactone core resulted in improved binding to the M. tuberculosis FabB (mtFabB; KasA) in vitro. ${ }^{33,} 52,53$ Here, we describe the synthesis, in vitro inhibition and in vivo efficacy of enantiopure (5R)-TLM analogs. These compounds were evaluated for activity against mtFabB, the $S$. aureus FabF enzyme (saFabF) and clinically relevant bacteria such as Mycobacterium tuberculosis, methicillin sensitive $S$. aureus (MSSA), MRSA, Francisella tularensis, Klebsiella pneumoniae, Burkholderia pseudomallei and Yersinia pestis. Collectively the studies provide additional data to direct the optimization of TLM.

\section{RESULTS AND DISCUSSION}

\section{Chemistry}

With the synthesis of the $\mathrm{C} 4$ analogs as a starting point, we followed literature precedent for the asymmetric synthesis of ( $5 R)$-TLM where the key dienyl oxathiolanone building block (32) was synthesized from D-alanine (Scheme 1) ${ }^{44}$ Preparation of $\mathbf{3 2}$ proceeded via the formation of a diastereomeric mixture of an oxathiolanone intermediate from which the required $2 R, 4 S$ diastereomer was isolated by crystallization from n-pentane at $-78^{\circ} \mathrm{C}$ and verified by $2 \mathrm{D}$ NMR. The oxathiolanone ring of $\mathbf{3 2}$ was subsequently opened using treatment with cesium carbonate in ethanol at $10{ }^{\circ} \mathrm{C}$ to release the unstable thiol (1). This was immediately acylated with selected acid chlorides to give the respective acylated derivatives 1a-4a, 12a-16a and 19a (80-90\% two-step yield). Subsequent enolate formation of 1a-4a, 12a-16a and 19a with LiHMDS at $-78^{\circ} \mathrm{C}$ initiated the thio-Dieckmann condensation providing (5R)-TLM-TLM 4, (5R)-TLM 12-16 and (5R)-TLM 19 in 58$92 \%$ yields, respectively (Scheme 2). Subsequent elaboration at the $\mathrm{C} 4$ position was performed using the TLM 2 scaffold, since alkylation of TLM was unsuccessful due to the formation of a mixture of C4, C2 O-alkylation, and C3 alkylation products. Preferential Oalkylation of TLM 2 was achieved using NaH in DMF with tert-butyl bromoester and followed by hydrolysis of the ester with $25 \%$ TFA in $\mathrm{CH}_{2} \mathrm{Cl}_{2}$ to obtain compound 22a. 
Compound 22a was coupled with 2-azidoethanamine and 2-azidoethylcarbamate under coupling conditions to generate TLM 22 and TLM 23 in $70 \%$ and $66 \%$ yields, respectively. Finally, TLM 24 and TLM 25 were obtained by treatment of TLM 22 with 27 in the presence of $\mathrm{CuSO}_{4} .5 \mathrm{H}_{2} \mathrm{O}$ and sodium ascorbate to form the corresponding 'click' adducts in $80 \%$ and $88 \%$ yields, respectively (Scheme 3 ).

Similarly, 1 was acylated with 30 to give compounds 18a and 20a followed by Dieckmann cyclization to provide TLM 18 and TLM 20 which were further treated with $\mathbf{2 7}$ under 'click' conditions to provide TLM 21 and TLM 17, respectively (Scheme 4).

Utilizing a previously published protocol for C 3 acylation of tetronic acids, ${ }^{54}$ TLM 2 was treated with a variety of anhydrides or acid chlorides (5a-11a and 26a) in the presence of $\mathrm{Et}_{3} \mathrm{~N}$ and catalytic amount of DMAP in $\mathrm{CH}_{2} \mathrm{Cl}_{2}$ to form kinetically controlled O-acylated products. Subsequently, DMAP mediated transfer of the O-acyl group to C 3 of TLM over time led to the thermodynamically stable C3-acylated products, providing TLM 5-11 and 26 in $70 \%-85 \%$ yields (Scheme 5).

\section{In vitro activity}

Previously we quantified the inhibition of mtFabB and C171Q mtFabB by TLM analogs 118. ${ }^{33,52}$ Here we expand these studies by analyzing the inhibition of $\mathrm{mtFabB}$ as well as FabF and C164Q FabF from $S$. aureus, by additional C3 and C4 analogs (TLM 19-25). We also determined the whole cell antibacterial activity of TLM (1) and all TLM analogs (2-25) against M. tuberculosis, Y. pestis, F. tularensis, B. pseudomallei, MSSA and MRSA.

C4 substituents of demethyl TLM-Compounds TLM 22-25 were synthesized by attaching substituents to the C4 position of demethyl TLM (TLM 2), rather than TLM, as TLM 2 showed similar affinity for mtFabB compared to TLM. However, none of the analogs were found to bind to mtFabB (Table 2), which was confirmed using WaterLOGSY based competition data (data not shown). These data were mirrored in the poor MIC values observed for the $\mathrm{C} 4$ analogs against $M$. tuberculosis. Moreover, the $\mathrm{C} 4$ analogs showed poor whole cell activity for all bacterial strains tested (Table 2 and Table S2). Careful analysis of the crystallographic data of TLM and derivatives bound to $\mathrm{mtFabB}$ and $\mathrm{mtC} 171 \mathrm{QFabB}$ revealed that the $\mathrm{C} 4$ hydroxyl is involved in an extended water-mediated hydrogen bonding network that includes Thr315, which is conserved in FabB and FabF from different bacterial species (Figure S1). These data support the need for a hydrogen bonding group at $\mathrm{C} 4$ and suggest that the hydrogen bonding network is essential for the binding of TLM analogs to FabB.

C3 substituents with bulky side chains-Based on the structural and interligand NOE NMR data, analogs TLM 12-17, 19 and 21 were synthesized. Despite improvements in binding affinity, particularly for TLM 12 and $\mathbf{1 7},{ }^{33}$ no improvements were observed in the whole cell activity of these analogs against M. tuberculosis (Table 3). TLM 16 showed poor binding to saFabF, the only $\beta$-ketoacyl-ACP synthase in the $S$. aureus FASII elongation cycle (Table 4). However, this analog showed a 5-fold improvement in MIC against MSSA (16 $\mu \mathrm{g} / \mathrm{mL})$ and a 3 -fold improvement against MRSA ( $25 \mu \mathrm{g} / \mathrm{mL})$ compared to TLM (75 $\mu \mathrm{g} /$ 
$\mathrm{mL}$ ). Bulky aryl substituents at the $\mathrm{C} 3$ position of the thiolactone ring were tolerated by $F$. tularensis as the whole cell activity of the lead molecule was retained for the analogs TLM 12-16 with MIC values in the range of 2-3 $\mu \mathrm{g} / \mathrm{mL}$. None of the analogs showed improvements in MIC values against $Y$. pestis, M. tuberculosis and B. pseudomallei compared to TLM (Tables 3, 4 and S2).

C3 alkyl substituents-The loss of time-dependent inhibition of acyl mtFabB upon removal of the methyl group at the C3 position (TLM 2), observed by Kapilashrami et al., ${ }^{33}$ triggered our interest in developing SAR for C3-substituted TLMs. Therefore, we studied analogs with alkyl substituents of variable chain lengths at the C3 position (TLM 3, 4, 18, 20). While these analogs showed subtle changes in binding affinity to $\mathrm{mtFabB}$, the length of the alkyl substituent modulated the drug:target residence time $\left(\mathrm{t}_{R}=1 / \mathrm{k}_{\text {off }}\right)$ with optimal $\mathrm{t}_{R}$ observed for TLM 3 binding to the C171Q mtFabB acyl-enzyme mimic. ${ }^{33,}{ }^{52}$ While the MIC values of these compounds against $M$. tuberculosis were either similar to the MIC for TLM $(3 \mu \mathrm{g} / \mathrm{mL})$ or larger, the ethyl analog TLM 3 showed activity against a TLM resistant strain of H37Rv, ${ }^{29}$ with an MIC value of $2.5 \mu \mathrm{g} / \mathrm{mL}$. In addition, these analogs did not show improvement in whole cell activity against $Y$. pestis, B. pseudomallei and $S$. aureus (Table 3 and Table S2).

C3 acyl substituents-Analogs of TLM were also designed in which the substituent was linked to the $\mathrm{C} 3$ position of the ring via a ketone (TLM 5-11, 26) in an attempt to more closely mimic the diketo motif of malonyl-ACP. While all the TLM analogs with acyl substituents at $\mathrm{C} 3$ showed tighter binding in vitro to $\mathrm{mtFabB}$ and the acylenzyme mtFabB mimic, ${ }^{33}$ no improvement was observed in the whole cell activity against $M$. tuberculosis (Table 3). The lack of correlation between $\mathrm{K}_{\mathrm{i}}$ and MIC values could reflect issues either with drug uptake or efflux. ${ }^{40,43}$ TLM 5, $\mathbf{6}$ and $\mathbf{1 1}$ showed tighter binding to saFabF compared to the lead molecule (Table 4): TLM 5 had a 8-fold improvement in binding to both apo saFabF and C164Q saFabF, compared to TLM $(90-100 \mu \mathrm{M})$, with a binding affinity of 14.2 and $11.9 \mu \mathrm{M}$, respectively (Table 4). However, these changes in affinity did not translate into similar improvements in MIC with TLM 5 only showing a 2-fold improvement in MIC (32 $\mu \mathrm{g} / \mathrm{mL})$ compared to TLM $(75 \mu \mathrm{g} / \mathrm{mL})$ for MRSA. In contrast, TLM 9 and TLM 11, despite poor binding affinities, had a 150-fold improvement in whole cell activity against MSSA $(\mathrm{MIC}=0.5 \mu \mathrm{g} / \mathrm{mL})$ and a 75- and 37-fold improvement against MRSA $(1 \mu \mathrm{g} / \mathrm{mL}$ and 2 $\mu \mathrm{g} / \mathrm{mL}$, respectively) compared to TLM $(75 \mu \mathrm{g} / \mathrm{mL})$. TLM 26, with an azido hexanoyl substituent at C3, showed a 5-fold improvement in whole cell activity, with an MIC value of $16 \mu \mathrm{g} / \mathrm{mL}$ against MSSA and MRSA (Table 3). Finally, TLM 3 showed similar whole cell activity against $B$. pseudomallei $(32 \mu \mathrm{g} / \mathrm{mL}$ ) to the lead compound $(8 \mu \mathrm{g} / \mathrm{mL}$ ) (Table S2). No improvements in whole cell activity were observed against $Y$. pestis.

\section{In vivo activity in MRSA and $K$. pneumoniae infection models}

In their seminal work, Miyakawa et al. studied the pharmacokinetics and in vivo activity of TLM in animal models of $K$. pneumoniae and $S$. marcescens infection. ${ }^{27}$ These investigators observed that TLM was rapidly absorbed and gave protection against systemic infection caused by both pathogens despite poor whole cell activity (100-200 $\mu \mathrm{g} / \mathrm{mL})$. We consequently decided to test the in vivo efficacy of selected analogs against MRSA, one of 
the pathogens studied in this work, as well as $K$. pneumoniae. In agreement with previous reports, TLM together with most analogs tested showed no toxicity below $100 \mu \mathrm{g} / \mathrm{mL}$ against THP1 monocytic leukemia cells (Table S3). For systemic infection with MRSA we chose analogs TLM 5, TLM 6 and TLM 11 which have improved $K_{d}$ values for saFabF compared to TLM, and MIC values of 32, 62 and $2 \mu \mathrm{g} / \mathrm{mL}$ against MRSA compared to an MIC value of $75 \mu \mathrm{g} / \mathrm{mL}$ for TLM. Although animals treated with TLM, TLM 5 and TLM 11 showed similar survival times compared to the vehicle control, TLM 6 gave an average survival time of 3 days compared to a survival time of 5 days for treatment with vancomycin (Figure 2A). Subsequently, the $\mathrm{ED}_{50}$ of TLM 6 was determined to be $\sim 125 \mathrm{mg} / \mathrm{kg}$, compared to an $\mathrm{ED}_{50}$ of $<30 \mathrm{mg} / \mathrm{kg}$ for vancomycin (Figure $\mathrm{S} 2$ ).

We then evaluated the activity of TLM 3, TLM 5 and TLM 6 in a mouse model of systemic K. pneumoniae (KPC) infection (Figure 2B). While gentamicin, a clinically used aminoglycoside antibiotic (MIC $<1 \mu \mathrm{g} / \mathrm{mL})$, showed maximum efficacy with a mean survival time of 6.2 days, TLM 5 and 6 showed improved survival times of 4.6 and 5.1 days respectively, compared to 3.25 days for TLM. We tested the in vitro antibacterial activity of these analogs and all had MIC values of $>250 \mu \mathrm{g} / \mathrm{mL}$ in the cell based assay. Thus, similar to the studies by Miyakawa et al., there was a disconnect between the in vitro and in vivo efficacy of the TLM analogs.

\section{SUMMARY}

The natural product TLM is an inhibitor of the $\beta$-ketoacyl-ACP synthases in the bacterial FAS II pathway. To provide a platform for the optimization of TLM, we have synthesized two series of enantiomerically pure ( $5 R)$-TLM analogs, and have evaluated their in vitro and in vivo antibacterial activity. These efforts have focused on the relatively underexploited $\mathrm{C} 3$ and $\mathrm{C} 4$ positions of the thiolactone core. In general, the C4 analogs of TLM, showed poor biochemical and microbiological activity which we believe can be attributed to disruption of a hydrogen bonding network that involves the TLM C4 hydroxyl group. ${ }^{52}$ Thus, elaboration of the thiolactone core will likely require the introduction of substituents that maintain interactions with this H-bonding network. In contrast, several of the C3 analogs had improved activity compared to the parent compound. TLM 11, with a bulky biphenylhexanoyl substituent at $\mathrm{C} 3$, had a 13 -fold improvement in MIC $(0.21 \mu \mathrm{g} / \mathrm{mL})$ against $F$. tularensis compared to TLM $(2.8 \mu \mathrm{g} / \mathrm{mL})$. Moreover, TLM 11 demonstrated 150- and 37fold improvement in MIC values against MSSA $(0.5 \mu \mathrm{g} / \mathrm{mL})$ and MRSA $(2 \mu \mathrm{g} / \mathrm{mL})$ compared to TLM (75 $\mu \mathrm{g} / \mathrm{mL})$, respectively, while TLM 26, with a hexanoyl azide substituent, showed a 5-fold improvement in whole cell activity with an MIC value of 16 $\mu \mathrm{g} / \mathrm{mL}$ against both strains of $S$. aureus. The trifluoroacetyl analog TLM 6 had improved in vitro activity towards saFabF and MRSA compared to TLM, and showed improved survival in a systemic MRSA infection model. Finally, despite poor whole cell activity TLM 3, 5 and $\mathbf{6}$ showed improved mean survival times in a systemic $K$. pneumoniae infection model. While a more in depth analysis is required to elucidate the disparity between the in vitro and in vivo activities of our analogs against $K$. pneumoniae, one possibility is that the residence time of the inhibitors on the $K$. pneumoniae KAS enzyme(s) may play a role in the observed in vivo efficacy. Alternatively, the target(s) for TLM and the TLM analogs may be more 
vulnerable in vivo and/or one or more metabolites might be responsible for the observed activity. Experiments are underway to investigate the thermodynamics and kinetics of binding of these analogs to the $\beta$-ketoacyl-ACP synthases from this pathogen. Our data suggest that further modifications at the thiolactone $\mathrm{C} 3$ position may be a profitable source of additional TLM-based inhibitors.

\section{EXPERIMENTAL SECTION}

\section{General Procedure I}

Cesium carbonate $(1.6 \mathrm{mmol})$ was added to a solution of $\mathbf{3 2}(1.6 \mathrm{mmol})$ in $\mathrm{EtOH}(6.0 \mathrm{~mL})$ at $10{ }^{\circ} \mathrm{C}$. After all the starting material had been consumed ( $20 \mathrm{~min}$ by TLC), the mixture was poured into $\mathrm{NH}_{4} \mathrm{Cl}$ (sat.) $/ 1 \mathrm{~N} \mathrm{HCl}(15 \mathrm{~mL}, 3: 1)$ and extracted with $\mathrm{Et}_{2} \mathrm{O}(3 \times 20 \mathrm{~mL})$ and then water $(3 \times 20 \mathrm{~mL})$. The combined organics were dried $\left(\mathrm{MgSO}_{4}\right)$, filtered and evaporated to provide 1 . Subsequently, the residue was redissolved in $\mathrm{CH}_{2} \mathrm{Cl}_{2}(15 \mathrm{~mL})$ and cooled to $0{ }^{\circ} \mathrm{C}$ followed by addition of $\mathrm{Et}_{3} \mathrm{~N}(2.0 \mathrm{mmol})$ and the respective acid chloride (1.6 mmol). After $1 \mathrm{~h}, \mathrm{NH}_{4} \mathrm{Cl}$ (sat. $20 \mathrm{~mL}$ ) was added and the mixture was extracted with $\mathrm{CH}_{2} \mathrm{Cl}_{2}(3 \times 15 \mathrm{~mL})$. The combined organics were dried $\left(\mathrm{MgSO}_{4}\right)$, filtered and evaporated. Flash chromatography (5\% EtOAc/hex) gave the pure compounds with yields of 80-90\%.

\section{General Procedure II}

LiHMDS was added to a stirred solution of compounds (1a-4a and 12a-19a) (1.09 mmol) in THF $(16.4 \mathrm{~mL})$ at $-78{ }^{\circ} \mathrm{C}(1.8 \mathrm{mmol}, 1.0 \mathrm{M}$ in THF $)$ and the solution was allowed to slowly warm to $-5{ }^{\circ} \mathrm{C}$. The solution was then poured into $1 \mathrm{~N} \mathrm{HCl}(25 \mathrm{~mL})$ and extracted with EtOAc $(3 \times 15 \mathrm{~mL})$. The combined organics were dried $\left(\mathrm{MgSO}_{4}\right)$, filtered and evaporated. This crude mixture was dissolved in $\mathrm{NaHCO}_{3}$ (sat, $15 \mathrm{~mL}$ ) and stirred for 15 min and extracted with $\mathrm{Et}_{2} \mathrm{O}(3 \times 10 \mathrm{~mL})$. The aqueous layer was then acidified to $\mathrm{pH} 3(\mathrm{pH}$ paper) with $1 \mathrm{~N} \mathrm{HCl}$ and extracted with $\mathrm{Et}_{2} \mathrm{O}(3 \times 10 \mathrm{~mL})$ and $\mathrm{EtOAc}(2 \times 10 \mathrm{~mL})$. The combined organics were dried $\left(\mathrm{MgSO}_{4}\right)$, filtered and evaporated to provide TLM -TLM 4 and TLM 12 - TLM 19, which were further purified by flash chromatography to get obtain the pure compounds in $58-92 \%$ yield.

\section{General Procedure III}

$\mathrm{CuSO}_{4} .5 \mathrm{H}_{2} \mathrm{O}(1.0 \mathrm{mmol})$ and sodium ascorbate $(1.0 \mathrm{mmol})$ were added to a solution of TLM 18, TLM 20, or TLM 22 (1.0 mmol) and alkyne (27) $(1.0 \mathrm{mmol})$ in n-butanol/water $(1: 1,3 \mathrm{~mL})$ at room temperature. The reaction mixture was stirred at room temperature for 1-2 $\mathrm{h}$ then filtered and concentrated, and the crude compound purified with an ISCO combiflash to afford the corresponding click product.

\section{General Procedure IV}

Respective anhydride or acid chloride $(1.5 \mathrm{mmol}), \mathrm{Et}_{3} \mathrm{~N}(2 \mathrm{mmol})$ and DMAP $(0.4 \mathrm{mmol})$ were added to a stirred solution of TLM $2(1.0 \mathrm{mmol})$ in $\mathrm{CH}_{2} \mathrm{Cl}_{2}(3 \mathrm{~mL})$ at $0{ }^{\circ} \mathrm{C}$. The reaction mixture was stirred at room temperature for 2-8 h then extracted with $\mathrm{CH}_{2} \mathrm{Cl}_{2}(3 \times$ $10 \mathrm{~mL})$. The organic phase was then washed with $1 \mathrm{~N} \mathrm{HCl}(20 \mathrm{~mL})$ and dried $\left(\mathrm{MgSO}_{4}\right)$, filtered and evaporated to provide residue, which was purified by flash chromatography or using an ISCO combiflash. 


\section{Analytical Data}

(R)-4-hydroxy-3,5-dimethyl-5-((E)-2-methylbuta-1,3-dienyl)thiophen-2(5H)one (TLM): General Procedure II-TLM 80\%. $[\alpha]_{D}^{23}=+173.0(c 1.2, \mathrm{MeOH}) . \mathrm{Mp}$ 119-121 ${ }^{\circ} \mathrm{C}$. [(lit. $\left.\left.{ }^{44}[\alpha]_{D}^{24}=+174(c 0.6, \mathrm{MeOH}), \mathrm{Mp} 119.5-121{ }^{\circ} \mathrm{C}\right)\right] ;{ }^{1} \mathrm{H} \mathrm{NMR}(500 \mathrm{MHz}$, $\mathrm{CDCl}_{3}$ and 5\% $\left.\mathrm{CD}_{3} \mathrm{OD}\right): \delta 1.64,1.65(2 \mathrm{~s}, 6 \mathrm{H}), 1.73(\mathrm{~s}, 3 \mathrm{H}), 1.91(\mathrm{~s}, 3 \mathrm{H}), 4.96 .(\mathrm{d}, J=11.0$ $\mathrm{Hz}, 1 \mathrm{H}), 5.15(\mathrm{~d}, J=17.0 \mathrm{~Hz}, 1 \mathrm{H}), 5.53(\mathrm{~s}, 1 \mathrm{H}), 6.26(\mathrm{dd}, J=17.0,11.0 \mathrm{~Hz}, 1 \mathrm{H}) .{ }^{13} \mathrm{C}$ NMR (125 MHz, $\mathrm{CDCl}_{3}$ and $\left.5 \% \mathrm{CD}_{3} \mathrm{OD}\right) \delta 7.2,11.6,29.3,55.1,109.3,113.2,130.0,139.1$,

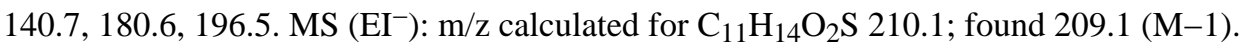

\section{(R,E)-Ethyl 2,4-dimethyl-2-(Acetylthio)hexa-3,5-dienoate (2a): General}

Procedure I-Flash chromatography (5\% EtOAc/hexanes) afforded 2a 84\%. $[\alpha]_{D}^{23}=+17$ (c 1.0, $\left.\mathrm{CH}_{2} \mathrm{Cl}_{2}\right) .{ }^{1} \mathrm{H}$ NMR (600 MHz, $\left.\mathrm{CDCl}_{3}\right): \delta 1.25,(\mathrm{t}, J=7.2 \mathrm{~Hz}, 3 \mathrm{H}), 1.84,1.87(2 \mathrm{~s}$, $6 \mathrm{H}), 1.21(\mathrm{~s}, 3 \mathrm{H}), 4.20 .(\mathrm{q}, J=7.2 \mathrm{~Hz}, 2 \mathrm{H}), 5.03(\mathrm{~d}, J=9.0 \mathrm{~Hz}, 1 \mathrm{H}), 5.20(\mathrm{~d}, J=14.5 \mathrm{~Hz}$, $1 \mathrm{H}), 5.73(\mathrm{~s}, 1 \mathrm{H}), 6.30(\mathrm{dd}, J=14.5,9.0 \mathrm{~Hz}, 1 \mathrm{H}) .{ }^{13} \mathrm{C} \mathrm{NMR}\left(125 \mathrm{MHz}, \mathrm{CDCl}_{3}\right) \delta 12.7$,

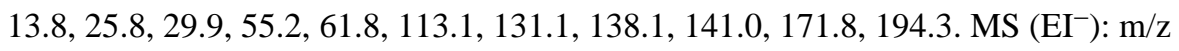
calculated for $\mathrm{C}_{12} \mathrm{H}_{18} \mathrm{O}_{3} \mathrm{~S} 242.1$; found $243.1(\mathrm{M}+1), 198.1(\mathrm{M}-44)$.

\section{(R)-4-hydroxy-5-methyl-5-((E)-2-methylbuta-1,3-dienyl)thiophen-2(5H)-one} (TLM 2): General Procedure II-TLM 2 58\%. $[\alpha]_{D}^{23}=+44(c 1.0 \mathrm{MeOH}) .\left[\left(\mathrm{lit}^{4}{ }^{47}[\alpha]_{D}^{29}\right.\right.$ $=+186(c 1.01, \mathrm{MeOH}), 1 \mathrm{H} \mathrm{NMR}\left(500 \mathrm{MHz}, \mathrm{CD}_{3} \mathrm{OD}\right): \delta 1.79(\mathrm{~s}, 3 \mathrm{H}), 1.83(\mathrm{~s}, 3 \mathrm{H}), 5.06$ (d, $J=10.5 \mathrm{~Hz}, 1 \mathrm{H}), 5.27$ (d, $J=17.7 \mathrm{~Hz}, 1 \mathrm{H}), 5.66(\mathrm{~s}, 1 \mathrm{H}), 6.36$ (dd, $J=17.5,10.5 \mathrm{~Hz}) .{ }^{13} \mathrm{C}$ NMR (100 MHz, $\left.\mathrm{CD}_{3} \mathrm{OD}\right): \delta 12.4,30.3,58.8,113.7,131.4,140.5,142.1,190.1,197.2$. MS $\left(\mathrm{EI}^{+}\right): \mathrm{m} / \mathrm{z}$ calculated for $\mathrm{C}_{10} \mathrm{H}_{12} \mathrm{O}_{2} \mathrm{~S} 196.1$; found $197.1(\mathrm{M}+1)$.

( $R, E$ )-Ethyl 2,4-dimethyl-2-(butanoylthio)hexa-3,5-dienoate (3a): General Procedure I-Flash chromatography (5\% EtOAc/hexanes) afforded the product 3a 82\%.

$[\alpha]_{D}^{23}=+32\left(c 1, \mathrm{CH}_{2} \mathrm{Cl}_{2}\right) .{ }^{1} \mathrm{H}$ NMR $\left(400 \mathrm{MHz}, \mathrm{CDCl}_{3}\right): \delta 0.88,(\mathrm{t}, J=7.2 \mathrm{~Hz}, 3 \mathrm{H}), 1.21(\mathrm{t}$, $J=7.2 \mathrm{~Hz}, 3 \mathrm{H}), 1.58-1.64(\mathrm{~m}, 2 \mathrm{H}), 1.82,1.84(2 \mathrm{~s}, 6 \mathrm{H}), 2.40(\mathrm{t}, J=7.2 \mathrm{~Hz}, 3 \mathrm{H}), 4.13-$ $4.18(\mathrm{~m}, 2 \mathrm{H}), 5.00(\mathrm{~d}, J=10.8 \mathrm{~Hz}, 1 \mathrm{H}), 5.20(\mathrm{~d}, J=17.2 \mathrm{~Hz}, 1 \mathrm{H}), 5.72(\mathrm{~s}, 1 \mathrm{H}), 6.30$ (dd, $J$ $=17.2,10.8 \mathrm{~Hz}, 1 \mathrm{H}) .{ }^{13} \mathrm{C} \mathrm{NMR}\left(100 \mathrm{MHz}, \mathrm{CDCl}_{3}\right) \delta 12.7,13.1,13.8,18.9,25.9,45.1$, 55.0, 61.8, 113.0, 131.2, 138.1, 141.0, 172.0, 198.0. MS $\left(\mathrm{EI}^{+}\right) \mathrm{m} / z$ calculated for $\mathrm{C}_{14} \mathrm{H}_{22} \mathrm{O}_{3} \mathrm{~S}$ 270.1; found $271.1(\mathrm{M}+\mathrm{H})$.

(R)-3-Ethyl 4-hydroxy-5-methyl-5-((E)-2-methylbuta-1,3dienyl)thiophen-2(5H)-one (TLM 3): General Procedure II-Flash chromatography (1:1, EtOAc/hexanes) afforded TLM $375 \% \cdot[\alpha]_{D}^{23}=+98(c 1, \mathrm{MeOH}) .{ }^{1} \mathrm{H}$ NMR $(500 \mathrm{MHz}$, $\mathrm{CDCl}_{3}$ and $\left.5 \% \mathrm{CD}_{3} \mathrm{OD}\right): \delta 1.97(\mathrm{t}, J=7.2 \mathrm{~Hz}, 3 \mathrm{H}), 1.68,1.77(2 \mathrm{~s}, 6 \mathrm{H}), 2.24(\mathrm{q}, J=7.2 \mathrm{~Hz}$, $2 \mathrm{H}), 4.98(\mathrm{~d}, J=10.5 \mathrm{~Hz}, 1 \mathrm{H}), 5.18(\mathrm{~d}, J=17.0 \mathrm{~Hz}, 1 \mathrm{H}), 5.53(\mathrm{~s}, 1 \mathrm{H}), 6.26$ (dd, $J=17.0$, $10.5 \mathrm{~Hz}, 1 \mathrm{H}) .{ }^{13} \mathrm{C} \mathrm{NMR}\left(125 \mathrm{MHz}, \mathrm{CDCl}_{3}\right.$ and $\left.5 \% \mathrm{CD}_{3} \mathrm{OD}\right) \delta 11.7,12.2,16.0,29.6,55.0$, 113.5, 115.3, 130.0, 139.4, 140.7, 180.6, 196.3. MS (EI') $\mathrm{m} / z$. 223.1 (M - 1). HRMS (M + $\mathrm{H})^{+} \mathrm{m} / \mathrm{z}$ calculated for $\mathrm{C}_{12} \mathrm{H}_{16} \mathrm{O}_{2} \mathrm{SH} 225.0949$; found 225.0949 . 
( $R, E)$-Ethyl 2,4-dimethyl-2-(pentanoylthio)hexa-3,5-dienoate (4a): General Procedure I-Flash chromatography (5\% EtOAc/hexanes) afforded 4a 84\%. ${ }^{1} \mathrm{H}$ NMR $\left(400 \mathrm{MHz}, \mathrm{CDCl}_{3}\right): \delta 0.85,(\mathrm{t}, J=7.2 \mathrm{~Hz}, 3 \mathrm{H}), 1.22(\mathrm{t}, J=7.2 \mathrm{~Hz}, 3 \mathrm{H}), 1.26-1.30(\mathrm{~m}, 2 \mathrm{H})$ $1.55-1.59(\mathrm{~m}, 2 \mathrm{H}), 1.82,1.84(2 \mathrm{~s}, 6 \mathrm{H}), 2.43(\mathrm{t}, J=7.2 \mathrm{~Hz}, 2 \mathrm{H}), 4.14-4.19(\mathrm{~m}, 2 \mathrm{H}), 5.00$ $(\mathrm{d}, J=10.8 \mathrm{~Hz}, 1 \mathrm{H}), 5.20(\mathrm{~d}, J=17.2 \mathrm{~Hz}, 1 \mathrm{H}), 5.72(\mathrm{~s}, 1 \mathrm{H}), 6.30(\mathrm{dd}, J=17.2,10.8 \mathrm{~Hz}, 1$ H). ${ }^{13} \mathrm{C}$ NMR $\left(100 \mathrm{MHz}, \mathrm{CDCl}_{3}\right) \delta 12.8,13.5,13.8,21.8,25.9,43.0,55.0,61.8,113.0$, 131.3, 131.5, 138.1, 141.2, 172.0, 198.2. MS $\left(\mathrm{EI}^{+}\right) \mathrm{m} / \mathrm{z}$ calculated for $\mathrm{C}_{15} \mathrm{H}_{24} \mathrm{O}_{3} \mathrm{~S} 284.1$; found $285.1(\mathrm{M}+1)$.

(R)-4-Hydroxy-5-methyl-5-((E)-2-methylbuta-1,3-dienyl)-3propylthiophen-2(5H)-one (TLM 4): General Procedure II-Flash chromatography (1:1, EtOAc/hexanes) afforded TLM 4 78\%. $[\alpha]_{D}^{23}=+61(c 1, \mathrm{MeOH}) .{ }^{1} \mathrm{H}$ NMR $(500 \mathrm{MHz}$, $\mathrm{CDCl}_{3}$ and $\left.5 \% \mathrm{CD}_{3} \mathrm{OD}\right): \delta 1.89(\mathrm{t}, J=7.2 \mathrm{~Hz}, 3 \mathrm{H}), 2.26-2.20(\mathrm{~m}, 2 \mathrm{H}), 1.73,1.85(2 \mathrm{~s}, 6$ H), 2.23 (q, $J=7.2 \mathrm{~Hz}, 2 \mathrm{H}), 5.02$. (d, $J=10.5 \mathrm{~Hz}, 1 \mathrm{H}), 5.22(\mathrm{~d}, J=17.0 \mathrm{~Hz}, 1 \mathrm{H}), 5.58$ (s, $1 \mathrm{H}), 6.27(\mathrm{dd}, J=17.0,10.5 \mathrm{~Hz}, 1 \mathrm{H}) .{ }^{13} \mathrm{C} \mathrm{NMR}\left(125 \mathrm{MHz}, \mathrm{CDCl}_{3}\right.$ and $\left.5 \% \mathrm{CD}_{3} \mathrm{OD}\right) \delta$ 12.0, 13.8, 21.1, 24.6, 29.8, 55.4, 113.6, 114.5, 129.3, 139.9, 140.7, 181.2, 197.5. MS (EI') $\mathrm{m} / \mathrm{z}$. $237.1(\mathrm{M}-1)$. HRMS $(\mathrm{M}+\mathrm{H})^{+} \mathrm{m} / z$ calculated for $\mathrm{C}_{13} \mathrm{H}_{18} \mathrm{O}_{2} \mathrm{SH} 239.1106$; found 239.1102 .

(R)-3-Acetyl-4-hydroxy-5-methyl-5-((E)-2-methylbuta-1,3dienyl)thiophen-2(5H)-one (TLM 5): General Procedure IV—Flash chromatography (30\% EtOAc/hexanes/1\% AcOH) afforded TLM 5 75\%. $[\alpha]_{D}^{23}=+404(c 1, \mathrm{MeOH}) .{ }^{1} \mathrm{H}$ NMR $\left(500 \mathrm{MHz}, \mathrm{CDCl}_{3}\right.$ and 5\% $\left.\mathrm{CD}_{3} \mathrm{OD}\right): \delta 1.68,1.79(2 \mathrm{~s}, 6 \mathrm{H}), 2.54(\mathrm{~s}, 3 \mathrm{H}), 5.04(\mathrm{~d}, J=$ $10.0 \mathrm{~Hz}, 1 \mathrm{H}), 5.20$ (d, $J=17.0 \mathrm{~Hz}, 1 \mathrm{H}), 5.64(\mathrm{~s}, 1 \mathrm{H}), 6.27$ (dd, $J=17.0,10.0 \mathrm{~Hz}, 1$ H). ${ }^{13} \mathrm{C}$ NMR (100 MHz, $\mathrm{CDCl}_{3}$ and $5 \% \mathrm{CD}_{3} \mathrm{OD}$, peak broadening was observed due to keto-enol tautomerism) $\delta 18.8,19.8,29.3,57.7,107.9,113.9,117.3,130.0,132.5,139.5$, 139.9, 193.9, 204.4. MS (EI $\left.{ }^{-}\right) \mathrm{m} / z .237 .2(\mathrm{M}-1)$. HRMS $(\mathrm{M}+\mathrm{H})^{+} \mathrm{m} / \mathrm{z}$ calculated for $\mathrm{C}_{12} \mathrm{H}_{14} \mathrm{O}_{3} \mathrm{SH} 239.0742$; found 239.0748 .

(R)-3-(2,2,2-Trifluoroacetyl)-4-hydroxy-5-methyl-5-((E)-2-methylbuta-1,3dienyl)thiophen-2(5H)-one (TLM 6): General Procedure IV—Flash chromatography (40\% hexanes $/ 10 \%$ THF/2\% AcOH/EtOAc) afforded TLM 6 82\%. $[\alpha]_{D}^{23}=+310(c 1$, MeOH). ${ }^{1} \mathrm{H}$ NMR (500 MHz, CD $\left.{ }_{3} \mathrm{OD}\right): \delta 1.72,1.75(2 \mathrm{~s}, 6 \mathrm{H}), 5.00$ (d, $\left.J=10.4 \mathrm{~Hz}, 1 \mathrm{H}\right)$, $5.20(\mathrm{~d}, J=17.6 \mathrm{~Hz}, 1 \mathrm{H}), 5.72(\mathrm{~s}, 1 \mathrm{H}), 6.37$ (dd, $J=17.6,10.4 \mathrm{~Hz}, 1 \mathrm{H}) .{ }^{13} \mathrm{C}$ NMR $(100$ $\mathrm{MHz}, \mathrm{CD}_{3} \mathrm{OD}$, peak broadening was observed due to keto-enol tautomerism) $\delta 16.9,18.8$, 51.3, 104.0, 114.0, 118.1-126.7 (q), 138.5, 140.1, 140.54, 193.3, 195.8, 201.7. MS (EI') $\mathrm{m} / \mathrm{z}$. $291.1(\mathrm{M}-1)$. HRMS $(\mathrm{M}+\mathrm{Na})^{+} \mathrm{m} / z$ calculated for $\mathrm{C}_{12} \mathrm{H}_{11} \mathrm{~F}_{3} \mathrm{O}_{3} \mathrm{SNa} 315.0279$; found 315.0229 .

(R)-3-Butyryl 4-hydroxy-5-methyl-5-((E)-2-methylbuta-1,3dienyl)thiophen-2(5H)-one (TLM 7): General Procedure IV—Flash chromatography (20\% EtOAc/hexanes/1\% AcOH) afforded TLM 7 80\%. $[\alpha]_{D}^{23}=+396(c 1, \mathrm{MeOH}) .{ }^{1} \mathrm{H}$ NMR (400 MHz, CDCl 3 ): $\delta 1.00$ (t, $J=7.60 \mathrm{~Hz}, 3 \mathrm{H}), 1.69-1.79$ (m, $5 \mathrm{H}), 1.86$ (s, $3 \mathrm{H}$ ), 2.92-3.01 (m, 2 H), 5.08 (d, $J=10.8 \mathrm{~Hz}, 1 \mathrm{H}), 5.25$ (d, $J=17.6 \mathrm{~Hz}, 1 \mathrm{H}), 5.69$ (s, $1 \mathrm{H}), 6.31$ 
(dd, $J=17.6,10.8 \mathrm{~Hz}, 1 \mathrm{H}) .{ }^{13} \mathrm{C}$ NMR $\left(100 \mathrm{MHz}, \mathrm{CDCl}_{3}\right) \delta 13.1,13.6,18.4,30.0,37.6$, 57.5, 108.0, 114.1, 130.0, 139.8, 140.2, 190.3, 198.3, 205.2. MS (EI-) $\mathrm{m} / z .265 .1(\mathrm{M}-1)$. HRMS $(\mathrm{M}+\mathrm{H})^{+} \mathrm{m} / z$ calculated for $\mathrm{C}_{14} \mathrm{H}_{18} \mathrm{O}_{3} \mathrm{SH} 267.1055$; found 267.1050 .

(R)-Methyl 2,5-dihydro-4-hydroxy-5-methyl-5-((E)-2-methylbuta-1,3-dienyl)-2oxothiophene-3-carboxylate (TLM 8): General Procedure IV-Flash

chromatography (3:1, Acetone/Toluene) afforded TLM $871 \% \cdot[\alpha]_{D}^{23}=+313$ (c 1, $\mathrm{MeOH}) .{ }^{1} \mathrm{H}$ NMR (400 MHz, CD $\left.\mathrm{OD}\right): \delta 1.48,1.51(2 \mathrm{~s}, 6 \mathrm{H}), 3.54$ (s, $\left.3 \mathrm{H}\right), 4.76(\mathrm{~d}, J=$ $10.8 \mathrm{~Hz}, 1 \mathrm{H}), 4.94(\mathrm{~d}, J=17.2 \mathrm{~Hz}, 1 \mathrm{H}), 5.45(\mathrm{~s}, 1 \mathrm{H}), 6.06(\mathrm{dd}, J=17.2,10.8 \mathrm{~Hz}, 1$ H). ${ }^{13} \mathrm{C}$ NMR $\left(100 \mathrm{MHz}, \mathrm{CD}_{3} \mathrm{OD}\right.$, peak broadening was observed due to keto-enol tautomerism) $\delta 20.4,23.8,52.3,56.3,98.5,113.3,134.2,139.7,142.4,170.7,182.3,206.2$. MS $\left(\mathrm{EI}^{-}\right) \mathrm{m} / \mathrm{z} .253 .1(\mathrm{M}-1), 195.1(\mathrm{M}-59)$. HRMS $(\mathrm{M}+\mathrm{Na})^{+} \mathrm{m} / \mathrm{z}$ calculated for $\mathrm{C}_{12} \mathrm{H}_{14} \mathrm{O}_{4} \mathrm{SNa}$ 277.0510; found 277.0513.

(R)-4-Hydroxy-5-methyl-5-((E)-2-methylbuta-1,3-dienyl)-3palmitoylthiophen-2(5H)-one (TLM 9): General Procedure IV—Flash

chromatography (1:1, EtOAc/hexanes) to afford TLM 9 75\%. $[\alpha]_{D}^{23}=+130(c 1$, $\left.\mathrm{CH}_{2} \mathrm{Cl}_{2}\right) .{ }^{1} \mathrm{H}$ NMR $\left(400 \mathrm{MHz}, \mathrm{CDCl}_{3}\right): \delta 0.87(\mathrm{t}, J=6.80 \mathrm{~Hz}, 3 \mathrm{H}), 1.80-1.31(\mathrm{~m}, 20 \mathrm{H})$, 1.40-1.59 (m, $4 \mathrm{H}), 1.69,1.71(2 \mathrm{~s}, 6 \mathrm{H}), 2.31(\mathrm{t}, J=6.80 \mathrm{~Hz}, 2 \mathrm{H}), 2.89$ (q, $J=7.20 \mathrm{~Hz}, 2$ H), $5.03(\mathrm{~d}, J=10.8 \mathrm{~Hz}, 1 \mathrm{H}), 5.18(\mathrm{~d}, J=17.6 \mathrm{~Hz}, 1 \mathrm{H}), 5.55(\mathrm{~s}, 1 \mathrm{H}), 6.27(\mathrm{dd}, J=17.6$, $10.8 \mathrm{~Hz}, 1 \mathrm{H}) .{ }^{13} \mathrm{C} \mathrm{NMR}\left(100 \mathrm{MHz}, \mathrm{CDCl}_{3}\right) \delta 12.8,14.0,22.6,246,25.0,28.9,29.2,29.4$, 29.6, 29.70, 29.73, 31.0, 31.9, 34.0, 40.0, 56.6, 108.7, 131.6, 139.5, 140.5, 190.3, 201.0, 204.6. MS $\left(\mathrm{EI}^{+}\right) \mathrm{m} / \mathrm{z} .435 .2(\mathrm{M}+1)$. HRMS $(\mathrm{M}+\mathrm{H})^{+} \mathrm{m} / \mathrm{z}$ calculated for $\mathrm{C}_{26} \mathrm{H}_{42} \mathrm{O}_{3} \mathrm{SH}$ 435.2933; found 435.2929.

(R)-3-(2-Carboxybenzoyl)-4-hydroxy-5-methyl-5-((E)-2-methylbuta-1,3dienyl)thiophen-2(5H)-one (TLM 10): General Procedure IV—Flash chromatography (2:1, Acetone/Toluene) to afford TLM $1070 \% \cdot[\alpha]_{D}^{23}=+243$ (c 1, MeOH). ${ }^{1} \mathrm{H}$ NMR (500 MHz, CD $\left.{ }_{3} \mathrm{OD}\right): \delta 1.77,1.78(2 \mathrm{~s}, 6 \mathrm{H}), 4.96$ (d, $\left.J=10.8 \mathrm{~Hz}, 1 \mathrm{H}\right)$, $5.17(\mathrm{~d}, J=17.5 \mathrm{~Hz}, 1 \mathrm{H}), 5.78(\mathrm{~s}, 1 \mathrm{H}), 6.32(\mathrm{dd}, J=17.5,10.8 \mathrm{~Hz}, 1 \mathrm{H}), 7.10(\mathrm{~d}, J=6.0$ $\mathrm{Hz}, 1 \mathrm{H}), 7.35-7.04$ (m, $2 \mathrm{H}$ ), 7.94 (br s, $1 \mathrm{H}) .{ }^{13} \mathrm{C} \mathrm{NMR}\left(125 \mathrm{MHz}, \mathrm{CD}_{3} \mathrm{OD}\right.$, peak broadening was observed due to keto-enol tautomerism) $\delta 20.4,49.0,58.8,110.3,113.1$, 127.4, 129.1, 130.7, 131.3, 134.8, 139.6, 173.8, 195.7, 196.8, 206.0. MS (EI-) m/z. 343.0 $(\mathrm{M}-1)$. HRMS $(\mathrm{M}+\mathrm{Na})^{+} \mathrm{m} / \mathrm{z}$ calculated for $\mathrm{C}_{18} \mathrm{H}_{16} \mathrm{O}_{5} \mathrm{SNa} 367.0616$; found 367.0612.

(R)-3-(4-Phenyl-phenylhexanoyl)-4-hydroxy-5-methyl-5-((E)-2-methylbuta-1,3dienyl)thiophen-2(5H)-one (TLM 11): General Procedure IV—Flash

chromatography (20\% EtOAc/hexanes/1\% AcOH) afforded TLM $1172 \% \cdot[\alpha]_{D}^{23}=+211(c$ 1, MeOH). ${ }^{1} \mathrm{H}$ NMR (400 MHz, $\mathrm{CDCl}_{3}$ ): $\delta 1.29-1.39$ (m, $\left.3 \mathrm{H}\right), 1.56-1.62(\mathrm{~m}, 3 \mathrm{H}), 1.68$, $1.72(2 \mathrm{~s}, 6 \mathrm{H}), 2.56-2.61(\mathrm{~m}, 2 \mathrm{H}), 2.79-2.85(\mathrm{~m}, 2 \mathrm{H}), 4.94(\mathrm{~d}, J=10.0 \mathrm{~Hz}, 1 \mathrm{H}), 5.14(\mathrm{~d}, J$ $=17.6 \mathrm{~Hz}, 1 \mathrm{H}), 5.07(\mathrm{~s}, 1 \mathrm{H}), 6.28(\mathrm{dd}, J=17.6,10 \mathrm{~Hz}, 1 \mathrm{H}), 7.15-7.53(\mathrm{~m}, 9 \mathrm{H}) .{ }^{13} \mathrm{C}$ NMR (100 MHz, $\mathrm{CDCl}_{3}$, peak broadening was observed due to keto-enol tautomerism) $\delta$ 13.2, 14.0, 21.0, 29.2, 29.6, 31.4, 35.4, 60.9, 126.8, 128.6, 138.4, 140.8, 141.5, 190.33, 
198.37, 205.0. MS (EI $\left.{ }^{+}\right) \mathrm{m} / z .447 .2(\mathrm{M}+1), 469.2(\mathrm{M}+\mathrm{Na}) . \operatorname{HRMS}(\mathrm{M}+\mathrm{H})^{+} \mathrm{m} / \mathrm{z}$ calculated for $\mathrm{C}_{28} \mathrm{H}_{30} \mathrm{O}_{3} \mathrm{SH} 447.1994$; found 447.1989 .

(R,E)-Ethyl 2-(4-chloro-phenylbutanoylthio)-2,4-dimethylhexa-3,5-dienoate (12a): General Procedure I-Flash chromatography (8\% EtOAc/hexanes) afforded 12a $85 \% .[\alpha]_{D}^{23}=-12\left(c 1, \mathrm{CH}_{2} \mathrm{Cl}_{2}\right) .{ }^{1} \mathrm{H}$ NMR $\left(400 \mathrm{MHz}, \mathrm{CDCl}_{3}\right): \delta 1.42,(\mathrm{t}, J=7.2 \mathrm{~Hz}, 3 \mathrm{H})$, 1.88, $1.90(2 \mathrm{~s}, 6 \mathrm{H}), 2.29$ (m, $2 \mathrm{H}), 2.75(\mathrm{t}, J=7.2 \mathrm{~Hz}, 2 \mathrm{H}), 2.83-2.90(\mathrm{~m}, 2 \mathrm{H}), 4.38$. (q, $J=$ $7.2 \mathrm{~Hz}, 2 \mathrm{H}), 5.22$ (d, $J=10.8 \mathrm{~Hz}, 1 \mathrm{H}), 5.38$ (d, $J=17.2 \mathrm{~Hz}, 1 \mathrm{H}), 5.94(\mathrm{~s}, 1 \mathrm{H}), 6.50$ (dd, $J$ $=17.2,10.8 \mathrm{~Hz}, 1 \mathrm{H}), 7.23-7.28(\mathrm{~m}, 4 \mathrm{H}) .{ }^{13} \mathrm{C} \mathrm{NMR}\left(125 \mathrm{MHz}, \mathrm{CDCl}_{3}\right) \delta 13.8,26.7,28.8$, $34.5,42.2,52.8,55.1,61.9,113.2,128.3,128.9,129.8,131.7,139.7,140.2,140.5,172.8$, 197.6. MS $\left(\mathrm{EI}^{+}\right) \mathrm{m} / \mathrm{z}$ calculated for $\mathrm{C}_{20} \mathrm{H}_{25} \mathrm{ClO}_{3} \mathrm{~S} 380.1$; found $381.0(\mathrm{M}+1)$.

(R)-3-(4-Chlorophenethyl)-4-hydroxy-5-methyl-5-((E)-2-methylbuta-1,3dienyl)thiophen-2(5H)-one (TLM 12). General Procedure II-Purified by flash chromatography (30\% EtOAc/hexanes) afforded TLM 12 86\%. $[\alpha]_{D}^{23}=+104$ ( $c 1$, $\left.\mathrm{CH}_{2} \mathrm{Cl}_{2}\right) .{ }^{1} \mathrm{H}$ NMR $\left(400 \mathrm{MHz}, \mathrm{CDCl}_{3}\right): \delta 1.69 .1 .81(2 \mathrm{~s}, 6 \mathrm{H}), 2.54-2.59(\mathrm{~m}, 2 \mathrm{H}), 2.74-$ $2.80(\mathrm{~m}, 2 \mathrm{H}) 5.07(\mathrm{~d}, J=11.0 \mathrm{~Hz}, 1 \mathrm{H}), 5.24(\mathrm{~d}, J=17.0 \mathrm{~Hz}, 1 \mathrm{H}), 5.54(\mathrm{~s} 1 \mathrm{H}), 6.28$ (dd, $J$ $=17.0,11.0 \mathrm{~Hz}, 1 \mathrm{H}), 7.14(\mathrm{~d}, J=8.0 \mathrm{~Hz}, 1 \mathrm{H}), 7.21(\mathrm{~d}, J=8.0 \mathrm{~Hz}, 1 \mathrm{H}) 7.23(\mathrm{~s}, 1 \mathrm{H}) .{ }^{13} \mathrm{C}$ NMR $\left(125 \mathrm{MHz}, \mathrm{CDCl}_{3}\right) \delta 12.0,24.4,29.7,32.8,55.51,113.4,113.9,128.3,128.94$,

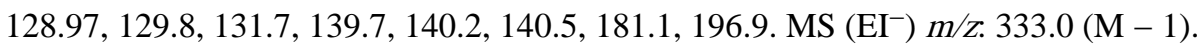
HRMS $(\mathrm{M}+\mathrm{H})^{+} \mathrm{m} / \mathrm{z}$ calculated for $\mathrm{C}_{18} \mathrm{H}_{19} \mathrm{ClO}_{2} \mathrm{SH} 335.0873$; found 335.0867 .

(R,E)-Ethyl 2-(4-methyl-phenylbutanoylthio)-2,4-dimethylhexa-3,5-dienoate (13a): General Procedure I-Flash chromatography (7\% EtOAc/hexanes) afforded 13a 80\%. $[\alpha]_{D}^{23}=-212\left(c 1, \mathrm{CH}_{2} \mathrm{Cl}_{2}\right) .{ }^{1} \mathrm{H}$ NMR $\left(500 \mathrm{MHz}, \mathrm{CDCl}_{3}\right): \delta 1.28,(\mathrm{t}, J=7.5 \mathrm{~Hz}, 3 \mathrm{H})$, 1.88, $1.90(2 \mathrm{~s}, 6 \mathrm{H}), 1.20-1.93(\mathrm{~m}, 2 \mathrm{H}), 2.33(\mathrm{~s}, 3 \mathrm{H}), 2.25(\mathrm{t}, J=7.5 \mathrm{~Hz}, 2 \mathrm{H}), 2.61(\mathrm{t}, J=$ $7.5 \mathrm{~Hz}, 2 \mathrm{H}), 4.23$. (q, $J=7.2 \mathrm{~Hz}, 2 \mathrm{H}), 5.07$ (d, $J=10.5 \mathrm{~Hz}, 1 \mathrm{H}), 5.24$ (d, $J=17.5 \mathrm{~Hz}, 1$ H), 5.79 (s, $1 \mathrm{H}), 6.35$ (dd, $J=17.5,10.5 \mathrm{~Hz}, 1 \mathrm{H}), 7.06-7.19$ (m, $4 \mathrm{H}) .{ }^{13} \mathrm{C}$ NMR $(125$ $\left.\mathrm{MHz}, \mathrm{CDCl}_{3}\right) \delta$ 13.0, 14.0, 20.1, 26.0, 27.1, 34.3, 42.6, 55.2, 113.2, 128.3, 129.0, 131.4, 135.4, 138.0, 138.2, 141.0, 172.1, 198.1. MS (EI $\left.{ }^{+}\right) \mathrm{m} / z$ calculated for $\mathrm{C}_{21} \mathrm{H}_{28} \mathrm{O}_{3} \mathrm{~S} 360.2$; found $361.1(\mathrm{M}+1)$.

(R)-3-(4-Methylphenethyl)-4-hydroxy-5-methyl-5-((E)-2-methylbuta-1,3dienyl)thiophen-2(5H)-one (TLM 13): Procedure II-Flash chromatography (30\% EtOAc/hexanes) afforded TLM 13 87\%. $[\alpha]_{D}^{23}=+161\left(c 1, \mathrm{CH}_{2} \mathrm{Cl}_{2}\right) .{ }^{1} \mathrm{H}$ NMR $(500 \mathrm{MHz}$, $\left.\mathrm{CDCl}_{3}\right): \delta 1.70,1.74(2 \mathrm{~s}, 6 \mathrm{H}), 2.31(\mathrm{~s}, 3 \mathrm{H}), 2.47-2.55(\mathrm{~m}, 1 \mathrm{H}), 2.60-2.54(\mathrm{~m}, 1 \mathrm{H}), 2.68-$ $2.78(\mathrm{~m}, 2 \mathrm{H}), 5.05(\mathrm{~d}, J=10.5 \mathrm{~Hz}, 1 \mathrm{H}), 5.24(\mathrm{~d}, J=17.5 \mathrm{~Hz}, 1 \mathrm{H}), 5.50(\mathrm{~s}, 1 \mathrm{H}), 6.30(\mathrm{dd}, J$ $=17.5,10.5 \mathrm{~Hz}, 1 \mathrm{H}), 7.03-7.12(\mathrm{~m}, 4 \mathrm{H}) .{ }^{13} \mathrm{C} \mathrm{NMR}\left(125 \mathrm{MHz}, \mathrm{CDCl}_{3}\right) \delta 12.1,21.0,25.2$, 29.7, 33.3, 55.0, 113.8, 113.9, 128.3, 129.14, 129.16, 129.3, 135.8, 138.4, 140.1, 140.6, 179.7, 195.4. MS $\left(\mathrm{EI}^{+}\right) \mathrm{m} / \mathrm{z} .315 .1(\mathrm{M}+1)$. HRMS $(\mathrm{M}+\mathrm{H})^{+} \mathrm{m} / \mathrm{z}$ calculated for $\mathrm{C}_{19} \mathrm{H}_{22} \mathrm{O}_{2} \mathrm{SH} 315.1419$; found 315.1411 .

(R,E)-Ethyl 2-(4-fluorophenyl-hexanoylthio)-2,4-dimethylhexa-3,5-dienoate (14a): General Procedure I-Flash chromatography (8\% EtOAc/hexanes) afforded 14a 
89\%. $[\alpha]_{D}^{23}=+965\left(c 1, \mathrm{CH}_{2} \mathrm{Cl}_{2}\right) .{ }^{1} \mathrm{H}$ NMR $\left(500 \mathrm{MHz}, \mathrm{CDCl}_{3}\right): \delta 1.24,(\mathrm{t}, J=7.5 \mathrm{~Hz}, 3 \mathrm{H})$, 1.26-1.34 (m, $2 \mathrm{H}), 1.35-1.67$ (2m, $4 \mathrm{H}), 1.85,1.87$ (2s, $6 \mathrm{H}), 2.42-2.55$ (m, $2 \mathrm{H}), 2.56-$ 2.59 (m, $2 \mathrm{H}), 4.20$. (q, $J=7.2 \mathrm{~Hz}, 2 \mathrm{H}), 5.03(\mathrm{~d}, J=9.0 \mathrm{~Hz}, 1 \mathrm{H}), 5.20(\mathrm{~d}, J=14.5 \mathrm{~Hz}, 1 \mathrm{H})$, $5.76(\mathrm{~s}, 1 \mathrm{H}), 6.32(\mathrm{dd}, J=14.5,9.0 \mathrm{~Hz}, 1 \mathrm{H}), 6.93-6.95(\mathrm{~m}, 2 \mathrm{H}), 7.08-7.10(\mathrm{~m}, 2 \mathrm{H}) .{ }^{13} \mathrm{C}$ NMR $\left(125 \mathrm{MHz}, \mathrm{CDCl}_{3}\right) \delta 12.9,13.9,25.7,25.9,28.6,30.9,34.7,43.1,55.1,113.1,114.8$,

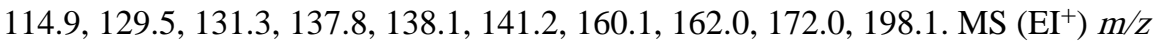
calculated for $\mathrm{C}_{22} \mathrm{H}_{29} \mathrm{FO}_{3} \mathrm{~S} 392.2$; found $393.1(\mathrm{M}+1)$, $410.2\left(\mathrm{M}+\mathrm{NH}_{4}\right)$.

(R)-3-(4-(4-Fluorophenyl)butyl)-4-hydroxy-5-methyl-5-((E)-2-methylbuta-1,3dienyl)thiophen-2(5H)-one (TLM 14): General Procedure II-Flash chromatography (25\% EtOAc/hexanes) afforded TLM 14 82\%. $[\alpha]_{D}^{23}=+63\left(c 1, \mathrm{CH}_{2} \mathrm{Cl}_{2}\right) \cdot{ }^{1} \mathrm{H}$ NMR (300 $\left.\mathrm{MHz}, \mathrm{CDCl}_{3}\right) \delta$ 1.50-1.61 (2m, $\left.4 \mathrm{H}\right), 1.70(\mathrm{~s}, 3 \mathrm{H}), 1.83(\mathrm{~s}, 3 \mathrm{H}), 2.26(\mathrm{t}, J=7.2 \mathrm{~Hz}, 2 \mathrm{H}$ ), $2.56(\mathrm{t}, J=7.2 \mathrm{~Hz}, 2 \mathrm{H}), 5.06(\mathrm{~d}, J=10.5 \mathrm{~Hz}, 1 \mathrm{H}), 5.24(\mathrm{~d}, J=17.5 \mathrm{~Hz}, 1 \mathrm{H}), 5.54(\mathrm{~s}, 1 \mathrm{H})$ 6.27 (dd, $J=17.5,10.5 \mathrm{~Hz}, 1 \mathrm{H}), 6.89-6.95$ (m, $2 \mathrm{H}), 7.05-7.10$ (m, $2 \mathrm{H}) .{ }^{13} \mathrm{C}$ NMR $(125$ $\left.\mathrm{MHz}, \mathrm{CDCl}_{3}\right) \delta 12.0,22.3,27.3,29.7,31.2,34.7,55.6,114.7,114.9,129.5,129.6,138.0$, 140.0, 140.6, 159.9, 162.3, 181.3, 197.7. ${ }^{19} \mathrm{~F}$ NMR (400 MHz, $\left.\mathrm{CDCl}_{3}\right) \delta-122.32(\mathrm{~m}) . \mathrm{MS}$ $\left(\mathrm{EI}^{+}\right) \mathrm{m} / \mathrm{z} .347 .1(\mathrm{M}+1)$. HRMS $(\mathrm{M}+\mathrm{H})^{+} \mathrm{m} / \mathrm{z}$ calculated for $\mathrm{C}_{20} \mathrm{H}_{23} \mathrm{FO}_{2} \mathrm{SH} 347.1481$; found 347.1478 .

(R,E)-Ethyl 2-(4-chlorophenyl-hexanoylthio)-2,4-dimethylhexa-3,5-dienoate (15a): General Procedure I-Flash chromatography (8\% EtOAc/hexanes) afforded 15a $85 \% .[\alpha]_{D}^{23}=-10(c 1, \mathrm{MeOH}) .{ }^{1} \mathrm{H} \mathrm{NMR}\left(500 \mathrm{MHz}, \mathrm{CDCl}_{3}\right): \delta 1.27,(\mathrm{t}, J=7.5 \mathrm{~Hz}, 3 \mathrm{H})$, 1.33-1.37 (m, 2 H), 1.61-1.68 (2m, 4 H), 1.87, 1.89 (2s, 6 H), 2.49 (t, $J=7.5 \mathrm{~Hz}, 2 \mathrm{H})$, 2.56-2.60 (m, $2 \mathrm{H}), 4.20$. (q, $J=7.5 \mathrm{~Hz}, 2 \mathrm{H}), 5.06(\mathrm{~d}, J=10.5 \mathrm{~Hz}, 1 \mathrm{H}), 5.23(\mathrm{~d}, J=17.5$ $\mathrm{Hz}, 1 \mathrm{H}), 5.78$ (s, $1 \mathrm{H}), 6.34$ (dd, $J=17.5,10.5 \mathrm{~Hz}, 1 \mathrm{H}), 7.09-7.10$ (m, $2 \mathrm{H}), 7.24-7.28$ (m, $2 \mathrm{H}) .{ }^{13} \mathrm{C} \mathrm{NMR}\left(125 \mathrm{MHz}, \mathrm{CDCl}_{3}\right) \delta 12.9,13.9,25.2,25.9,28.1,30.7,34.8,43.1,55.1$, 113.1, 128.2, 128.3, 129.5, 129.6, 131.2, 131.3, 138.1, 141.6, 141.2, 172.0, 198.1. MS (EI $\left.{ }^{+}\right)$ $\mathrm{m} / \mathrm{z}$ calculated for $\mathrm{C}_{22} \mathrm{H}_{29} \mathrm{ClO}_{3} \mathrm{~S} 408.2$; found $409.1(\mathrm{M}+1), 426.2\left(\mathrm{M}+\mathrm{NH}_{4}\right)$.

(R)-3-(4-(4-Chlorophenyl)butyl)-4-hydroxy-5-methyl-5-((E)-2-methylbuta-1,3dienyl)thiophen-2(5H)-one (TLM 15): General Procedure II-Purified by flash

chromatography (20\% EtOAc/hexanes) afforded TLM 15 83\%. $[\alpha]_{D}^{23}=+48(c 1$, $\mathrm{MeOH}) .{ }^{1} \mathrm{H}$ NMR $\left(500 \mathrm{MHz}, \mathrm{CDCl}_{3}\right) \delta 1.50-1.62(2 \mathrm{~m}, 4 \mathrm{H}), 1.73(\mathrm{~s}, 3 \mathrm{H}), 1.85(\mathrm{~s}, 3 \mathrm{H})$, 2.28 (t, $J=7.2 \mathrm{~Hz}, 2 \mathrm{H}), 2.56$ (t, $J=7.2 \mathrm{~Hz}, 2 \mathrm{H}), 5.06$ (d, $J=10.5 \mathrm{~Hz}, 1 \mathrm{H}), 5.26(\mathrm{~d}, J=$ $17.5 \mathrm{~Hz}, 1 \mathrm{H}), 5.56$ (s, $1 \mathrm{H}), 6.28$ (dd, $J=17.5,10.5 \mathrm{~Hz}, 1 \mathrm{H}), 7.07-7.09$ (m, $2 \mathrm{H}), 7.22-7.25$ (m, $2 \mathrm{H}) .{ }^{13} \mathrm{C} \mathrm{NMR}\left(125 \mathrm{MHz}, \mathrm{CDCl}_{3}\right) \delta 12.0,22.4,27.2,30.2,31.0,34.9,55.9,114.2$, 114.8, 128.3-129.6 (m), 131.3, 140.0, 140.4, 140.7, 179.8, 198.2. MS (EI+) m/z. $363.1(\mathrm{M}$ $+1)$. HRMS $(\mathrm{M}+\mathrm{H})^{+} \mathrm{m} / z$ calculated for $\mathrm{C}_{20} \mathrm{H}_{23} \mathrm{ClO}_{2} \mathrm{SH} 363.1186$; found 363.1180 .

( $R, E)$-Ethyl 2-(4-phenyl-phenyl-hexanoylthio)-2,4-dimethylhexa-3,5-dienoate (16a): General Procedure I-Purified by flash chromatography (7\% EtOAc/hexanes) to form (16a) $87 \%$. $[\alpha]_{D}^{23}=-158\left(c 1, \mathrm{CH}_{2} \mathrm{Cl}_{2}\right) .{ }^{1} \mathrm{H} \mathrm{NMR}\left(500 \mathrm{MHz}, \mathrm{CDCl}_{3}\right): \delta 1.27,(\mathrm{t}, J=$ $7.5 \mathrm{~Hz}, 3 \mathrm{H}), 1.36-1.48(\mathrm{~m}, 2 \mathrm{H}), 1.59-1.72(2 \mathrm{~m}, 4 \mathrm{H}), 1.89,1.91(2 \mathrm{~s}, 6 \mathrm{H}), 2.52(\mathrm{t}, J=7.5$ $\mathrm{Hz}, 2 \mathrm{H}), 2.65-2.69$ (m, $2 \mathrm{H}), 4.24$. (q, $J=7.5 \mathrm{~Hz}, 2 \mathrm{H}), 5.07$ (d, $J=10.5 \mathrm{~Hz}, 1 \mathrm{H}), 5.25$ (d, $J$ 
$=17.5 \mathrm{~Hz}, 1 \mathrm{H}), 5.80(\mathrm{~s}, 1 \mathrm{H}), 6.35(\mathrm{dd}, J=17.5,10.5 \mathrm{~Hz}, 1 \mathrm{H}), 7.25-7.61(\mathrm{~m}, 9 \mathrm{H}) .{ }^{13} \mathrm{C}$

NMR (125 MHz, $\left.\mathrm{CDCl}_{3}\right) \delta 12.9,13.9,25.3,25.9,28.4,30.9,35.2,43.2,55.2,113.2,126.9-$ $128.7(\mathrm{~m}), 131.4,138.2,138.6,141.2,141.4,172.1,198.2 . \mathrm{MS}\left(\mathrm{EI}^{+}\right) \mathrm{m} / z$ calculated for $\mathrm{C}_{28} \mathrm{H}_{34} \mathrm{O}_{3} \mathrm{~S} 450.2$; found $451.2(\mathrm{M}+1), 468.2\left(\mathrm{M}+\mathrm{NH}_{4}\right)$.

(R)-3-(4-(4-Phenylphenyl)butyl)-4-hydroxy-5-methyl-5-((E)-2-methylbuta-1,3dienyl)thiophen-2(5H)-one (TLM 16): General Procedure II-Flash chromatography (20\% EtOAc/hexanes) afforded TLM 16 86\%. $[\alpha]_{D}^{23}=-91\left(c 1, \mathrm{CH}_{2} \mathrm{Cl}_{2}\right) .{ }^{1} \mathrm{H}$ NMR (500 $\left.\mathrm{MHz}, \mathrm{CDCl}_{3}\right) \delta 1.50-1.69(2 \mathrm{~m}, 4 \mathrm{H}), 1.75(\mathrm{~s}, 3 \mathrm{H}), 1.86(\mathrm{~s}, 3 \mathrm{H}), 2.32(\mathrm{t}, J=7.2 \mathrm{~Hz}, 2 \mathrm{H})$, $2.67(\mathrm{t}, J=7.2 \mathrm{~Hz}, 2 \mathrm{H}), 5.08(\mathrm{~d}, J=10.5 \mathrm{~Hz}, 1 \mathrm{H}), 5.26(\mathrm{~d}, J=17.5 \mathrm{~Hz}, 1 \mathrm{H}), 5.57(\mathrm{~s}, 1 \mathrm{H})$, $6.30(\mathrm{dd}, J=17.5,10.5 \mathrm{~Hz}, 1 \mathrm{H}), 7.23-7.59(\mathrm{~m}, 9 \mathrm{H}) .{ }^{13} \mathrm{C} \mathrm{NMR}\left(125 \mathrm{MHz}, \mathrm{CDCl}_{3}\right) \delta 12.0$, 22.5, 27.4, 30.2, 31.1, 35.2, 55.0, 114.1, 114.8, 126.9-128.8 (m), 138.6, 140.5, 140.6, 141.0, 141.6, 179.4, 195.9. MS $\left(\mathrm{EI}^{+}\right) \mathrm{m} / \mathrm{z} .405 .1(\mathrm{M}+1)$. HRMS $(\mathrm{M}+\mathrm{H})^{+} \mathrm{m} / \mathrm{z}$ calculated for $\mathrm{C}_{26} \mathrm{H}_{28} \mathrm{O}_{2} \mathrm{SH} 405.1888$; found 405.1895 .

4-((4-Fluorophenylsulfonyl)amino)- $N-((1-(4-((R)-2,5-d i h y d r o-4-h y d r o x y-5-$ methyl-5-((E)-2-methylbuta-1,3-dienyl)-2-oxothiophen-3-yl)butyl)-1 H-1,2,3triazol-4-yl)methyl)benzamide (TLM 17): General Procedure III-Chromatography on silica gel (ISCO combiflash, $4 \mathrm{~g}$ gold cartridge, eluting with $\mathrm{CH}_{2} \mathrm{Cl}_{2} \rightarrow 8 \% \mathrm{MeOH}$ in $\left.\mathrm{CH}_{2} \mathrm{Cl}_{2}\right)$ afforded TLM 17 75\%. $[\alpha]_{D}^{23}=+70(c 1, \mathrm{MeOH}) .{ }^{1} \mathrm{H}$ NMR $\left(400 \mathrm{MHz}, \mathrm{CD}_{3} \mathrm{OD}\right): \delta$ 1.39-1.43 (m, 2 H), 1.67, 1.76 (2 s, 6 H), 1.89-1.92 (2 s, 2 H), 2.20-2.29 (m, 2 H), 4.32$4.42(\mathrm{~m}, 2 \mathrm{H}), 4.56-4.62(\mathrm{~m}, 2 \mathrm{H}), 5.03(\mathrm{~d}, J=10.8, \mathrm{~Hz}, 1 \mathrm{H}), 5.25(\mathrm{~d}, J=17.6 \mathrm{~Hz}, 1 \mathrm{H})$, $5.58(\mathrm{~s}, 1 \mathrm{H}), 6.37$ (dd, $J=17.6,10.8 \mathrm{~Hz}, 1 \mathrm{H}), 7.18-7.24(\mathrm{~m}, 4 \mathrm{H}), 7.69-7.78(\mathrm{~m}, 2 \mathrm{H})$, 7.84-7.87 (m, $2 \mathrm{H}) .{ }^{13} \mathrm{C}$ NMR (100 MHz, $\left.\mathrm{CDCl}_{3}\right) \delta 20.4,20.5$, 22.7, 25.6, 36.1, 50.3, 55.6, 113.6, 117.1, 117.3, 120.3, 123.5, 129.5, 131.0, 131.1, 132.1, 131.3, 137.0, 139.7, 141.4, 142.0, 165.0, 166.6, 167.0, 193.6, MS (EI+) $\mathrm{m} / \mathrm{z} .626 .1(\mathrm{M}+1), 627.2(\mathrm{M}+2)$. HRMS (M + $\mathrm{H})^{+} \mathrm{m} / \mathrm{z}$ calculated for $\mathrm{C}_{30} \mathrm{H}_{32} \mathrm{FN}_{5} \mathrm{O}_{5} \mathrm{~S}_{2} \mathrm{H} 626.1907$; found 626.1910 .

( $R, E)$-Ethyl 2-(6-azido-hexanoylthio)-2,4-dimethylhexa-3,5-dienoate (18a): General Procedure I-Flash chromatography (7\% EtOAc/hexanes) afforded 18a 85\%.

$[\alpha]_{D}^{23}=+6\left(c 1, \mathrm{CH}_{2} \mathrm{Cl}_{2}\right) .{ }^{1} \mathrm{H}$ NMR $\left(500 \mathrm{MHz}, \mathrm{CDCl}_{3}\right) \delta 1.23(\mathrm{t}, J=7.2 \mathrm{~Hz}, 3 \mathrm{H}), 1.39-1.34$ $(\mathrm{m}, 2 \mathrm{H}), 1.58-1.52(\mathrm{~m}, 2 \mathrm{H}), 1.67-1.60(\mathrm{~m}, 2 \mathrm{H}) 1.85,1.83(2 \mathrm{~s}, 6 \mathrm{H}), 2.46(\mathrm{t}, J=7.5 \mathrm{~Hz}, 2$ H), $3.22(\mathrm{t}, J=6.5 \mathrm{~Hz}, 2 \mathrm{H}), 4.18-4.16(\mathrm{~m}, 2 \mathrm{H}), 5.00(\mathrm{~d}, J=11.0 \mathrm{~Hz}, 1 \mathrm{H}), 5.20(\mathrm{~d}, J=17.5$ $\mathrm{Hz}, 1 \mathrm{H}), 5.71$ (s, $1 \mathrm{H}), 6.30$ (dd, $J=17.5,10.8 \mathrm{~Hz}, 1 \mathrm{H}) .{ }^{13} \mathrm{C} \mathrm{NMR}\left(125 \mathrm{MHz}, \mathrm{CDCl}_{3}\right) \delta$ 12.90, 13.90, 24.9, 25.8, 25.9, 28.4, 43.0, 51.0, 55.2, 61.9, 113.2, 131.2, 131.3, 138.1, 141.1, 172.0, 197.9. MS $\left(\mathrm{EI}^{+}\right) \mathrm{m} / \mathrm{z}$ calculated for $\mathrm{C}_{16} \mathrm{H}_{25} \mathrm{~N}_{3} \mathrm{O}_{3} \mathrm{~S} 339.2$; found $340.1(\mathrm{M}+1)$.

(R)-3-(4-Azidobutyl)-4-hydroxy-5-methyl-5-((E)-2-methylbuta-1,3dienyl)thiophen-2(5H)-one (TLM 18): General Procedure II-Flash chromatography (30\% EtOAc/hexanes) afforded TLM 18 75\%. $[\alpha]_{D}^{23}=+106\left(c 1, \mathrm{CH}_{2} \mathrm{Cl}_{2}\right) .{ }^{1} \mathrm{H}$ NMR $(400$ $\left.\mathrm{MHz}, \mathrm{CDCl}_{3}\right): \delta 1.21(\mathrm{t}, J=7.5 \mathrm{~Hz}, 3 \mathrm{H}), 1.73,1.85(2 \mathrm{~s}, 6 \mathrm{H}), 2.26(\mathrm{~m}, 2 \mathrm{H}), 2.30-2.28(\mathrm{~m}$, $2 \mathrm{H}), 3.33(\mathrm{t}, J=6.5 \mathrm{~Hz}, 2 \mathrm{H}), 5.03 .(\mathrm{d}, J=10.0 \mathrm{~Hz}, 1 \mathrm{H}), 5.22(\mathrm{~d}, J=17.0 \mathrm{~Hz}, 1 \mathrm{H}), 5.54(\mathrm{~s}$, $1 \mathrm{H}), 6.27$ (dd, $J=17.0,10.0 \mathrm{~Hz}, 1 \mathrm{H}) .{ }^{13} \mathrm{C} \mathrm{NMR}\left(100 \mathrm{MHz}, \mathrm{CDCl}_{3}\right) \delta 12.6,21.9,25.2$, 


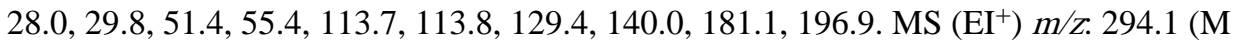
$+1)$. HRMS $(\mathrm{M}+\mathrm{H})^{+} \mathrm{m} / z$ calculated for $\mathrm{C}_{14} \mathrm{H}_{19} \mathrm{~N}_{3} \mathrm{O}_{2} \mathrm{SH} 294.1276$; found 294.1281.

(R,E)-Ethyl 2-(4-bromophenyl-hexanoylthio)-2,4-dimethylhexa-3,5-dienoate (19a): General Procedure I-Flash chromatography (8\% EtOAc/hexanes) afforded 19a $80 \% .[\alpha]_{D}^{23}=-70(c 1, \mathrm{MeOH}) .{ }^{1} \mathrm{H}$ NMR $\left(500 \mathrm{MHz}, \mathrm{CDCl}_{3}\right): \delta 1.27,(\mathrm{t}, J=7.5 \mathrm{~Hz}, 3 \mathrm{H})$, 1.30-1.36 (m, $2 \mathrm{H}), 1.59-1.68(2 \mathrm{~m}, 4 \mathrm{H}), 1.87,1.89(2 \mathrm{~s}, 6 \mathrm{H}), 2.49$ (t, $J=7.5 \mathrm{~Hz}, 2 \mathrm{H})$, $2.52-2.60$ (m, $2 \mathrm{H}), 4.20$. (q, $J=7.5 \mathrm{~Hz}, 2 \mathrm{H}), 5.06(\mathrm{~d}, J=10.5 \mathrm{~Hz}, 1 \mathrm{H}), 5.24$ (d, $J=17.5$ $\mathrm{Hz}, 1 \mathrm{H}), 5.78$ (s, $1 \mathrm{H}), 6.34$ (dd, $J=17.5,10.5 \mathrm{~Hz}, 1 \mathrm{H}), 7.04-7.06$ (m, $2 \mathrm{H}), 7.39-7.41$ (m, $2 \mathrm{H}) .{ }^{13} \mathrm{C} \mathrm{NMR}\left(125 \mathrm{MHz}, \mathrm{CDCl}_{3}\right) \delta 12.9,13.9,25.2,25.9,28.2,30.7,35.0,43.2,55.2$, 113.2, 119.3, 130.0-131.4 (m), 138.2, 141.6, 172.0, 198.2. MS $\left(\mathrm{EI}^{+}\right) \mathrm{m} / \mathrm{z}$ calculated for $\mathrm{C}_{22} \mathrm{H}_{29} \mathrm{BrO}_{3} \mathrm{~S} 452.1$; found 453.1 and $455.1(\mathrm{M}+1), 470.1$ and $472.1\left(\mathrm{M}+\mathrm{NH}_{4}\right)$.

(R)-3-(4-(4-Bromophenyl)butyl)-4-hydroxy-5-methyl-5-((E)-2-methylbuta-1,3dienyl)thiophen-2(5H)-one (TLM 19): General Procedure II-Flash chromatography (20\% EtOAc/hexanes) afforded TLM 19 78\%. $[\alpha]_{D}^{23}=+57\left(c 1, \mathrm{CH}_{2} \mathrm{Cl}_{2}\right) .{ }^{1} \mathrm{H}$ NMR (500 $\left.\mathrm{MHz}, \mathrm{CDCl}_{3}\right) \delta 1.50-1.64(2 \mathrm{~m}, 4 \mathrm{H}), 1.73(\mathrm{~s}, 3 \mathrm{H}), 1.85(\mathrm{~s}, 3 \mathrm{H}), 2.28(\mathrm{t}, J=7.2 \mathrm{~Hz}, 2 \mathrm{H})$, $2.56(\mathrm{t}, J=7.2 \mathrm{~Hz}, 2 \mathrm{H}), 5.09(\mathrm{~d}, J=10.5 \mathrm{~Hz}, 1 \mathrm{H}), 5.26(\mathrm{~d}, J=17.5 \mathrm{~Hz}, 1 \mathrm{H}), 5.56(\mathrm{~s}, 1 \mathrm{H})$, 6.29 (dd, $J=17.5,10.5 \mathrm{~Hz}, 1 \mathrm{H}), 7.02-7.04$ (m, $2 \mathrm{H}), 7.37-7.39$ (m, $2 \mathrm{H}) .{ }^{13} \mathrm{C}$ NMR $(125$ $\left.\mathrm{MHz}, \mathrm{CDCl}_{3}\right) \delta 12.0,21.0,27.2,30.2,31.0,34.9,55.0,114.1,114.7,119.3,128.8-130.1$ (m), 131.3, 140.4, 140.6, 141.4, 179.5, 195.8. MS $\left(\mathrm{EI}^{+}\right) \mathrm{m} / \mathrm{z} .407 .0(\mathrm{M}+1)$. HRMS (M + $\mathrm{H})^{+} \mathrm{m} / \mathrm{z}$ calculated for $\mathrm{C}_{20} \mathrm{H}_{23} \mathrm{BrO}_{2} \mathrm{SH} 407.0680$; found 407.0670.

(R,E)-Ethyl 2-(5-azido-pentanoylthio)-2,4-dimethylhexa-3,5-dienoate (20a): General Procedure I-Flash chromatography (7\% EtOAc/hexanes) afforded 20a 90\%. $[\alpha]_{D}^{23}=-3.6\left(c 1, \mathrm{CH}_{2} \mathrm{Cl}_{2}\right) .{ }^{1} \mathrm{H} \mathrm{NMR}\left(500 \mathrm{MHz}, \mathrm{CDCl}_{3}\right) \delta 1.22(\mathrm{t}, J=7.2 \mathrm{~Hz}, 3 \mathrm{H}), 1.55-$ $1.58(\mathrm{~m}, 2 \mathrm{H}), 1.67-1.70(\mathrm{~m}, 2 \mathrm{H}) 1.82,1.84(2 \mathrm{~s}, 6 \mathrm{H}), 2.46-2.49(\mathrm{~m}, 2 \mathrm{H}), 3.23(\mathrm{t}, J=6.5$ $\mathrm{Hz}, 2 \mathrm{H}), 4.14-4.19$ (m, $2 \mathrm{H}), 5.00$ (d, $J=11.0 \mathrm{~Hz}, 1 \mathrm{H}), 5.20$ (d, $J=17.5 \mathrm{~Hz}, 1 \mathrm{H}), 5.71$ (s, $1 \mathrm{H}), 6.30(\mathrm{dd}, J=17.5,10.8 \mathrm{~Hz}, 1 \mathrm{H}) .{ }^{13} \mathrm{C}$ NMR $\left(125 \mathrm{MHz}, \mathrm{CDCl}_{3}\right) \delta 12.85,13.8,25.9$ 27.8, 42.4, 50.8, 55.2, 61.9, 113.2, 131.13, 131.16, 138.2, 141.0, 172.0, 197.5. MS (EI $\left.{ }^{+}\right) \mathrm{m} / \mathrm{z}$ calculated $\mathrm{C}_{15} \mathrm{H}_{23} \mathrm{~N}_{3} \mathrm{O}_{3} \mathrm{~S} 325.2$; found $326.0(\mathrm{M}+1)$.

(R)-3-(3-Azidopropyl)-4-hydroxy-5-methyl-5-((E)-2-methylbuta-1,3dienyl)thiophen-2(5H)-one (TLM 20): General Procedure II-Flash chromatography (30\% EtOAc/hexanes) afforded TLM 20 75\%. $[\alpha]_{D}^{23}=+13.6$ (c 1, MeOH). ${ }^{1} \mathrm{H}$ NMR (500 $\left.\mathrm{MHz}, \mathrm{CD}_{3} \mathrm{OD}\right): \delta 1.21(\mathrm{t}, J=7.5 \mathrm{~Hz}, 2 \mathrm{H}), 1.71,1.78(2 \mathrm{~s}, 6 \mathrm{H}), 2.26(\mathrm{~m}, 2 \mathrm{H}), 3.26(\mathrm{~m}, 2$ H), 5.02. (d, $J=10.0 \mathrm{~Hz}, 1 \mathrm{H}), 5.20(\mathrm{~d}, J=17.0 \mathrm{~Hz}, 1 \mathrm{H}), 5.54(\mathrm{~s}, 1 \mathrm{H}), 6.27(\mathrm{dd}, J=17.0$, $10.0 \mathrm{~Hz}, 1 \mathrm{H}) .{ }^{13} \mathrm{C}$ NMR $(125 \mathrm{MHz}, \mathrm{MeOH}-\mathrm{d} 4) \delta 19.4,26.6,29.254 .7,112.1,113.0,114.5$, 129.3, 139.2, 140.3, 181.6, 195.9. MS (EI $\left.{ }^{-}\right) \mathrm{m} / z .278 .0(\mathrm{M}-1)$. HRMS $(\mathrm{M}+\mathrm{H})^{+} \mathrm{m} / \mathrm{z}$ calculated for $\mathrm{C}_{13} \mathrm{H}_{17} \mathrm{~N}_{3} \mathrm{O}_{2} \mathrm{SH} 280.1120$; found 280.1121 .

(2R)-N-((1-(4-((R)-2,5-Dihydro-4-hydroxy-5-methyl-5-((E)-2-methylbuta-1,3dienyl)-2-oxothiophen-3-yl)butyl)-1 H-1,2,3-triazol-4-yl)methyl)-2,4dihydroxy-3,3-dimethylbutanamide (TLM 21): General Procedure III- 
Chromatography on silica gel (ISCO combiflash, 4 g gold cartridge, eluting with $\mathrm{CH}_{2} \mathrm{Cl}_{2} \rightarrow$ $8 \% \mathrm{MeOH}$ in $\left.\mathrm{CH}_{2} \mathrm{Cl}_{2}\right)$ afforded TLM 21 85\%. $[\alpha]_{D}^{23}=+82(c 1, \mathrm{MeOH}) .{ }^{1} \mathrm{H}$ NMR (400 $\mathrm{MHz}, \mathrm{CD}_{3} \mathrm{OD}$ ): $\delta 0.92$ (br s, $\left.6 \mathrm{H}\right), 1.43$ (br s, $\left.2 \mathrm{H}\right), 1.70,1.78(2 \mathrm{~s}, 6 \mathrm{H}), 1.86-1.91(\mathrm{~m}, 2 \mathrm{H})$, 2.24 (br s, $2 \mathrm{H}$ ), 3.38-3.52 (m, $2 \mathrm{H}), 3.95$ (br s, $1 \mathrm{H}$ ), 4.34-4.56 (m, $4 \mathrm{H}), 5.03$ (d, $J=10.8$, $\mathrm{Hz}, 1 \mathrm{H}), 5.25(\mathrm{~d}, J=17.6 \mathrm{~Hz}, 1 \mathrm{H}), 5.60(\mathrm{~s}, 1 \mathrm{H}), 6.37$ (dd, $J=17.6,10.8 \mathrm{~Hz}, 1 \mathrm{H}), 7.89$ (s, 1H). ${ }^{13} \mathrm{C} \mathrm{NMR}\left(100 \mathrm{MHz}, \mathrm{CDCl}_{3}\right) \delta 14.5,19.8,21.3,22.8,25.8,30.6,35.6$ 40.3, 51.1, 71.9,

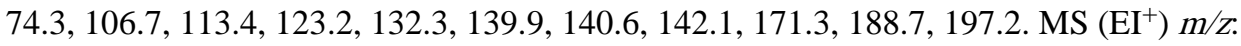
$461.2(\mathrm{M}-17), 479.3\left(\mathrm{M}+\mathrm{NH}_{4}\right)$. HRMS $(\mathrm{M}+\mathrm{H})^{+} \mathrm{m} / \mathrm{z}$ calculated for $\mathrm{C}_{23} \mathrm{H}_{34} \mathrm{~N}_{4} \mathrm{O}_{5} \mathrm{SH}$ 479.2328; found 479.2325 .

\section{2-((R)-2,5-Dihydro-2-methyl-2-((E)-2-methylbuta-1,3-dienyl)-5-oxothiophen-3- yloxy)- $\mathbf{N}$-(2-azidoethyl)acetamide (TLM 22)- $\mathrm{NaH}$ (60\% dispersed in oil) (148 mg,} $6.16 \mathrm{mmol})$ was added to a stirred solution of TLM $2(0.5 \mathrm{~g},(2.55 \mathrm{mmol})$ in DMF $(10 \mathrm{~mL})$ at room temperature and the mixture was stirred for a further $30 \mathrm{~min}$. tert-butyl bromoacetate $(0.75 \mathrm{~mL}, 5.10 \mathrm{mmol})$ was then added and the mixture stirred overnight. After completion, the reaction mixture was extracted with EtOAc $(3 \times 10 \mathrm{~mL})$ and the combined organics were dried $\left(\mathrm{MgSO}_{4}\right)$, filtered and evaporated. The crude material was purified by flash chromatography $\left(5 \% \mathrm{MeOH} / \mathrm{CH}_{2} \mathrm{Cl}_{2}\right.$ ) to obtain a O-alkylated TLM 2 derivative, which was redissolved in $\mathrm{CH}_{2} \mathrm{Cl}_{2}(10 \mathrm{~mL})$. TFA $(2.5 \mathrm{~mL})$ was added and the solvent was removed by evaporation after stirring for $24 \mathrm{~h}$ at room temperature. Purification by flash chromatography (1:1 EtOAc/hexanes/2\% $\mathrm{AcOH})$ to afford 22a in $90 \%$ yield. $\mathrm{MS}\left(\mathrm{EI}^{-}\right)$: $253.1(\mathrm{M}-1)$. $\mathrm{Et}_{3} \mathrm{~N}(287 \mu \mathrm{L}, 2 \mathrm{mmol})$ and $\mathrm{EDC}(230 \mathrm{mg}, 1.2 \mathrm{mmol})$ were added to a solution of 22a (254 mg, $1.0 \mathrm{mmol})$ and 2-azidoethanamine (103 mg, $1.2 \mathrm{mmol})$ in DMF (2 $\mathrm{mL}$ ) at room temperature and stirred overnight. After completion, the reaction mixture was extracted with $\mathrm{CH}_{2} \mathrm{Cl}_{2}(2 \times 10 \mathrm{~mL})$ and the combined organic phase was washed with $1 \mathrm{~N}$ $\mathrm{HCl}(10 \mathrm{~mL})$, dried $\left(\mathrm{MgSO}_{4}\right)$, filtered and evaporated to obtain the crude material, which was subsequently purified by flash chromatography (1:1, EtOAc/hexanes) to yield TLM 22 $70 \% \cdot[\alpha]_{D}^{23}=+131\left(c 1, \mathrm{CH}_{2} \mathrm{Cl}_{2}\right) \cdot{ }^{1} \mathrm{H}$ NMR $\left(400 \mathrm{MHz}, \mathrm{CDCl}_{3}\right): \delta 1.65,1.79(2 \mathrm{~s}, 6 \mathrm{H}), 3.34$ (br s, $4 \mathrm{H}), 4.41(\mathrm{~s}, 1 \mathrm{H}), 4.98(\mathrm{~d}, J=10.8 \mathrm{~Hz}, 1 \mathrm{H}), 5.15(\mathrm{~d}, J=17.2 \mathrm{~Hz}, 1 \mathrm{H}), 5.55(\mathrm{~s}, 1 \mathrm{H})$, 6.21 (dd, $J=17.2,10.8 \mathrm{~Hz}, 1 \mathrm{H}), 7.14(\mathrm{br} \mathrm{s}, 1 \mathrm{H}),{ }^{13} \mathrm{C} \mathrm{NMR}\left(100 \mathrm{MHz}, \mathrm{CDCl}_{3}\right) \delta 11.9$, 29.4, 38.4, 50.0, 57.0, 69.9, 102.1, 113.7, 128.82, 128.87, 139.5, 166.0, 184.7, 193.8. MS $\left(\mathrm{EI}^{+}\right) \mathrm{m} / \mathrm{z} .323 .1(\mathrm{M}+1), 345.1(\mathrm{M}+\mathrm{Na})$. HRMS $(\mathrm{M}+\mathrm{H})^{+} \mathrm{m} / \mathrm{z}$ calculated for $\mathrm{C}_{14} \mathrm{H}_{18} \mathrm{~N}_{4} \mathrm{O}_{3} \mathrm{SH} 323.1178$; found 323.1179.

\section{tert-Butyl-2-(2-((R)-2,5-dihydro-2-methyl-2-((E)-2-methylbuta-1,3-dienyl)-5- oxothiophen-3-yloxy)acetamido)ethylcarbamate (TLM 23)— $\mathrm{Et}_{3} \mathrm{~N}(287 \mu \mathrm{L}, 2$} $\mathrm{mmol}$ ) and EDC (230 mg, $1.2 \mathrm{mmol})$ were added to a solution of 22a $(254 \mathrm{mg}, 1.0 \mathrm{mmol})$ and tert-butyl 2-azidoethylcarbamate $(192 \mathrm{mg}, 1.2 \mathrm{mmol})$ in DMF $(4 \mathrm{~mL})$ at room temperature after which the solution was stirred overnight. After completion, the reaction mixture was extracted with $\mathrm{CH}_{2} \mathrm{Cl}_{2}(2 \times 10 \mathrm{~mL})$ and the combined organic phase was washed with $1 \mathrm{~N} \mathrm{HCl}(10 \mathrm{~mL})$, dried $\left(\mathrm{MgSO}_{4}\right)$, filtered and evaporated to obtain the crude product, which was then purified by flash chromatography (1:1, EtOAc/hexanes) to yield

TLM 23 in 66\% yield. $[\alpha]_{D}^{23}=-116\left(c 1, \mathrm{CH}_{2} \mathrm{Cl}_{2}\right) .{ }^{1} \mathrm{H} \mathrm{NMR}\left(500 \mathrm{MHz}, \mathrm{CDCl}_{3}\right): \delta 1.37$ (s, 9 H), 1.73, 1.89 (2 s, 6 H), 3.19-3.23 (m, 2 H), 3.31-3.42 (m, 2 H), 4.43 (s, 2 H), 5.04 (d, $J$ 
$=11.0 \mathrm{~Hz}, 1 \mathrm{H}), 5.08($ br s, $1 \mathrm{H}), 5.24(\mathrm{~d}, J=17.5 \mathrm{~Hz}, 1 \mathrm{H}), 5.55(\mathrm{~s}, 1 \mathrm{H}), 6.30(\mathrm{dd}, J=17.5$, $11.0 \mathrm{~Hz}, 1 \mathrm{H}), 7.18$ (br s, $1 \mathrm{H}),{ }^{13} \mathrm{C}$ NMR $\left(125 \mathrm{MHz}, \mathrm{CDCl}_{3}\right) \delta 28.1,29.5,39.9,40.7,57.0$, 70.3, 79.6, 102.4, 113.8, 129.3, 139.4, 140.5, 156.8, 165.8, 184.4, 193.0. MS $\left(\mathrm{EI}^{+}\right) \mathrm{m} / \mathrm{z}$. $397.1(\mathrm{M}+1)$. HRMS $(\mathrm{M}+\mathrm{H})^{+} \mathrm{m} / z$ calculated for $\mathrm{C}_{19} \mathrm{H}_{28} \mathrm{~N}_{2} \mathrm{O}_{5} \mathrm{SH} 397.1797$; found 397.1804 .

$(2 R)-N-((1-(2-(2-((R)-2,5-D i h y d r o-2-m e t h y l-2-((E)-2-m e t h y l b u t a-1,3-d i e n y l)-5-$ oxothiophen-3-yloxy)acetamido)ethyl)-1H-1,2,3-triazol-4-yl)methyl)-2,4dihydroxy-3,3-dimethylbutanamide (TLM 24): General Procedure IIIChromatography on silica gel (ISCO combiflash, $4 \mathrm{~g}$ gold cartridge, eluting with $\mathrm{CH}_{2} \mathrm{Cl}_{2} \rightarrow$ $\left.7 \% \mathrm{MeOH}_{\text {inCH }} \mathrm{Cl}_{2}\right)$ afforded TLM 24 in $80 \%$ yield. $[\alpha]_{D}^{23}=+94(c 1, \mathrm{MeOH}) .{ }^{1} \mathrm{H}$ NMR (500 MHz, $\left.\mathrm{CD}_{3} \mathrm{OD}, 10 \% \mathrm{CDCl}_{3}\right): \delta 1.05(\mathrm{~s}, 6 \mathrm{H}), 1.89,2.01(2 \mathrm{~s}, 6 \mathrm{H}), 3.40-3.49(\mathrm{~m}, 2 \mathrm{H})$, 3.73-3.77 (m, $1 \mathrm{H}), 3.95(\mathrm{~s}, 1 \mathrm{H}), 4.45-4.59(\mathrm{~m}, 8 \mathrm{H}), 5.08(\mathrm{~d}, J=10.75 \mathrm{~Hz}, 1 \mathrm{H}), 5.28(\mathrm{~d}, J$ $=17.5 \mathrm{~Hz}, 1 \mathrm{H}), 5.47(\mathrm{~s}, 1 \mathrm{H}), 5.77(\mathrm{~s}, 1 \mathrm{H}), 6.38(\mathrm{dd}, J=17.5,10.75 \mathrm{~Hz}, 1 \mathrm{H}), 7.78(\mathrm{br} \mathrm{s}, 1$ $\mathrm{H}), 8.30$ (s, $1 \mathrm{H}) .{ }^{13} \mathrm{C} \mathrm{NMR}\left(125 \mathrm{MHz}, \mathrm{CD}_{3} \mathrm{OD}, 10 \% \mathrm{CDCl}_{3}\right) \delta 11.5,14.3,14.5,19.8,20.2$, 28.8, 33.8, 39.1, 48.3, 57.3, 69.1, 70.0, 76.2, 101.9, 112.8, 123.2, 129.5, 139.2, 140.6, 167.2, 174.7, 186.0, 194.5. MS (EI-) $\mathrm{m} / \mathrm{z} .490 .2(\mathrm{M}-17), 508.2(\mathrm{M}+1)$. HRMS $(\mathrm{M}+\mathrm{H})^{+} \mathrm{m} / \mathrm{z}$ calculated for $\mathrm{C}_{23} \mathrm{H}_{33} \mathrm{~N}_{5} \mathrm{O}_{6} \mathrm{SH} 508.2230$; found 508.2230.

\section{$N-((1-(2-(2-((R)-2,5-D i h y d r o-2-m e t h y l-2-((E)-2-m e t h y l b u t a-1,3-d i e n y l)-5-$} oxothiophen-3-yloxy)acetamido)ethyl)-1 H-1,2,3-triazol-4-yl)methyl)-4-((4fluorophenylsulfonyl)-amino)benzamide (TLM 25): General Procedure IIIChromatography on silica gel (ISCO combiflash, 4 g gold cartridge, eluting with $\mathrm{CH}_{2} \mathrm{Cl}_{2} \rightarrow$ $7 \% \mathrm{MeOH}$ in $\left.\mathrm{CH}_{2} \mathrm{Cl}_{2}\right)$ afforded TLM 25 in $88 \%$ yield. $[\alpha]_{D}^{23}=+71(c 1, \mathrm{MeOH}) .{ }^{1} \mathrm{H} \mathrm{NMR}$ $\left(500 \mathrm{MHz}, \mathrm{CD}_{3} \mathrm{OD}, 10 \% \mathrm{CDCl}_{3}\right): \delta 1.75,1.85(2 \mathrm{~s}, 6 \mathrm{H}), 3.72-3.76(\mathrm{~m}, 2 \mathrm{H}), 4.50-4.58(\mathrm{~m}$, $8 \mathrm{H}), 5.07(\mathrm{~d}, J=10.5 \mathrm{~Hz}, 1 \mathrm{H}), 5.24(\mathrm{~d}, J=17.5 \mathrm{~Hz}, 1 \mathrm{H}), 5.41(\mathrm{~s}, 1 \mathrm{H}), 5.71(\mathrm{~s}, 1 \mathrm{H}), 6.30$ (d, $J=17.5,10.5 \mathrm{~Hz}, 1 \mathrm{H}), 7.13-7.19(\mathrm{~m}, 4 \mathrm{H}), 7.73(\mathrm{~m}, 1 \mathrm{H}), 7.83-7.85(\mathrm{~m}, 3 \mathrm{H}), 8.05(\mathrm{~s}, 1$ H). ${ }^{13} \mathrm{C}$ NMR (125 MHz, $\left.\mathrm{CD}_{3} \mathrm{OD}, 10 \% \mathrm{CDCl}_{3}\right) \delta 12.8,20.7,29.9,35.9,39.9,40.0,50.0$, $58.3,70.9,102.9,114.2,120.1,129.4,130.2,130.6,130.7,136.4,140.2,141.4,141.7$, 165.0, 167.1, 167.9, 168.0, 168.7, 186.8, 195.7. MS (EI $\left.{ }^{+}\right) \mathrm{m} / \mathrm{z} .655 .2(\mathrm{M}+1)$. HRMS (M + $\mathrm{H})^{+} \mathrm{m} / \mathrm{z}$ calculated for $\mathrm{C}_{30} \mathrm{H}_{31} \mathrm{FN}_{6} \mathrm{O}_{6} \mathrm{~S}_{2} \mathrm{H} 655.1809$; found 655.1812 .

(R)-3-(6-Azidohexanoyl)-4-hydroxy-5-methyl-5-((E)-2-methylbuta-1,3dienyl)thiophen-2(5H)-one (TLM 26): General Procedure IV—Flash

chromatography (30\% EtOAc/hexanes/1\% AcOH) afforded TLM $2685 \% \cdot[\alpha]_{D}^{23}=+183(c$ 1, MeOH). ${ }^{1} \mathrm{H}$ NMR (500 MHz, $\left.\mathrm{CDCl}_{3}\right) \delta 1.37-1.41(\mathrm{~m}, 2 \mathrm{H}), 1.56-1.65(\mathrm{~m}, 4 \mathrm{H}), 1.68$, $1.72(2 \mathrm{~s}, 6 \mathrm{H}), 2.72-2.90(\mathrm{~m}, 2 \mathrm{H}), 3.23-2.28(\mathrm{~m}, 2 \mathrm{H}), 5.03(\mathrm{~d}, J=10.5 \mathrm{~Hz}, 1 \mathrm{H}), 5.14(\mathrm{~d}, J$ $=17.5 \mathrm{~Hz}, 1 \mathrm{H}), 5.63(\mathrm{~s}, 1 \mathrm{H}), 6.28(\mathrm{dd}, J=17.6,10 \mathrm{~Hz}, 1 \mathrm{H}) .{ }^{13} \mathrm{C} \mathrm{NMR}\left(100 \mathrm{MHz}, \mathrm{CDCl}_{3}\right.$, peaks broadening was observed due to keto-enol tautomerism) $\delta 13.2,14.0,24.1,26.2,28.4$,

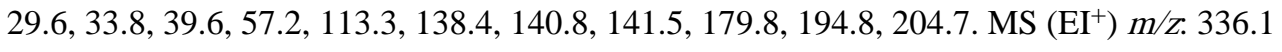
$(\mathrm{M}+1), 353.1\left(\mathrm{M}+\mathrm{NH}_{4}\right)$. HRMS $(\mathrm{M}+\mathrm{H})^{+} \mathrm{m} / z$ calculated for $\mathrm{C}_{16} \mathrm{H}_{21} \mathrm{~N}_{3} \mathrm{O}_{3} \mathrm{SH} 336.1382$; found 336.1375 . 
Materials and Methods-All commercially available chemicals and dry solvents (except THF which was distilled from sodium benzophenone ketyl) were purchased and used without further purification unless noted otherwise. Air- and moisture-sensitive reactions were run under inert atmosphere $\left(\mathrm{N}_{2}\right.$ or $\left.\mathrm{Ar}\right)$ using flame-dried glassware. ${ }^{1} \mathrm{H}$ and ${ }^{13} \mathrm{C}$ NMR spectra were acquired on a Varian and/or Bruker 400, 500 and $600 \mathrm{MHz}$ NMR spectrometer. Chemical shifts are expressed in parts per million ( $\delta$ value) downfield from tetramethylsilane using tetramethylsilane $(\delta=0)$ and/or relative to residual solvents such as $\mathrm{CHCl}_{3}\left(7.26 \mathrm{ppm},{ }^{1} \mathrm{H} ; 77.0 \mathrm{ppm},{ }^{13} \mathrm{C}\right), \mathrm{MeOH}-d_{4}\left(3.31 \mathrm{ppm},{ }^{1} \mathrm{H} ; 49.1 \mathrm{ppm},{ }^{13} \mathrm{C}\right)$, or acetone- $d_{6}\left(2.04 \mathrm{ppm},{ }^{1} \mathrm{H} ; 29.9 \mathrm{ppm},{ }^{13} \mathrm{C}\right)$. Splitting patterns are indicated as s, singlet; brs broad singlet; d, doublet; t, triplet; q, quartet; $\mathrm{m}$, multiplet; dt, doublet of triplet. TLC was performed on Merck pre-coated silica gel $60 \mathrm{~F}_{254}$ plates and all the spots were visualized using UV light followed by staining with phosphomolybdic acid. Column chromatography was performed using silica gel 60 (Merck, 230-400 mesh) and/or an ISCO Combiflash instrument using ISCO redisep flash cartridges (particle size: 35-70 $\mu \mathrm{m}$ ). Measurements of optical rotations were performed using a Perkin Elmer 241 automatic digital polarimeter. Mass spectra were obtained using electrospray (ES) ionization techniques. High-resolution mass spectra were obtained from the mass spectrometry laboratory at the University of Illinois, Urbana Champaign, Urbana, IL. TLM and TLM 2 analytical data was agreement with the reported data. ${ }^{44,}{ }^{47}$ All the C3 acylated (TLM 5-11 and 26) compounds showed extended keto-enol tautomerism, ${ }^{55}$ and peak broadening was generally observed in ${ }^{13} \mathrm{C}$ NMR Spectrum. The purity of all final compounds was determined to be $\$ 95 \%$ by reverse phase HPLC (Agilent HPLC, C-18 column, with $\mathrm{MeOH} / \mathrm{H}_{2} \mathrm{O}$ as the mobile phase).

Cloning and protein purification-The $S$. aureus fabFgene was cloned into a pET23a vector using KpnI and EcoRI restriction sites. The intrinsic NdeI cut site was removed using Quikchange mutagenesis and subsequently the gene was cloned into pET15b between NdeI and BamHI sites. Due to problems in obtaining soluble protein with the original construct, the I5K mutation was introduced into the NdeI cloning primer. Subsequently, the C165Q mutation was introduced using Quikchange mutagenesis. The primers used in this study are described in Table S1. The plasmids encoding mtFabB (pFPCA) $)^{32}$ and saFabF were transformed into electro-competent $M$. smegmatis $\mathrm{MC}^{2} 155$ and chemically competent $E$. coli BL21 (DE3) PlysS cell lines, respectively. The proteins were purified using standard affinity chromatography as described previously. ${ }^{32,33}$ Briefly, the cells were resuspended in $40 \mathrm{~mL}$ binding buffer $(20 \mathrm{mM}$ Tris- $\mathrm{HCl}, 800 \mathrm{mM} \mathrm{NaCl}, 5 \mathrm{mM}$ imidazole, $\mathrm{pH}$ 7.9) and lysed by sonication. The cellular debris was removed by ultracentrifugation at $40,000 \mathrm{rpm}$ for $1 \mathrm{~h}$ at $4{ }^{\circ} \mathrm{C}$, after which the supernatant was filtered and loaded onto a His-bind column. The column was then washed with $40 \mathrm{~mL}$ binding buffer, 80 $\mathrm{mL}$ wash buffer (20 mM Tris-HCl, $150 \mathrm{mM} \mathrm{NaCl}, 60 \mathrm{mM}$ imidazole, $\mathrm{pH}$ 7.9) and subsequently eluted ( $20 \mathrm{mM}$ Tris- $\mathrm{HCl}, 800 \mathrm{mM} \mathrm{NaCl}, 1 \mathrm{M}$ imidazole, $\mathrm{pH}$ 7.9). The fractions containing protein were pooled and loaded onto a Superdex 200 column equilibrated with $30 \mathrm{mM}$ Tris- $\mathrm{HCl}, 150 \mathrm{mM} \mathrm{NaCl}, \mathrm{pH}$ 8.5. The purified proteins were analyzed with SDS-PAGE. 
Direct Binding experiments-The binding kinetics and thermodynamics of select TLM analogs were conducted by monitoring quenching of intrinsic tryptophan fluorescence of mtFabB, C171Q-mtFabB, saFabF and C165Q-saFabF as described previously. ${ }^{33}$

MIC determination-Protocols followed for MIC determinations for B. pseudomallei, $Y$. pestis, F. tularensis, $S$. aureus (MSSA, MRSA), and $K$. pneumoniae were similar except for the growth media. Compounds were added to the 96-well plate starting at $512 \mu \mathrm{g} / \mathrm{mL}$ in the first column and serially diluted 1:2 to column 12 for a final concentration of $0.25 \mu \mathrm{g} / \mathrm{mL}$ in CAMHB (or MMH for F. tularensis). MIC plates were incubated at $37^{\circ} \mathrm{C}$ for $18-24 \mathrm{~h}$ at which time $10 \mu \mathrm{L}$ of Alamar Blue (Invitrogen, Carlsbad, California) was added to each well and plates were incubated for an additional $4 \mathrm{~h}$. Reduction of Alamar Blue was determined by absorbance readings at wavelengths of $570 \mathrm{~nm}$ and $600 \mathrm{~nm}$ using a microplate reader (Biotek, Winooski, VT). Percent growth reduction was calculated and $\mathrm{MIC}_{90}$ values were determined by plotting the percentage inhibition calculated from spectrophotometric readings over the drug concentration series. Bacterial growth and MIC values were confirmed by optical density. Non-linear regression analysis was run on $\%$ growth inhibition curves to determine $\mathrm{MIC}_{90}$ values.

A modified 96-well Alamar blue assay (MABA) used for the generation of MICs for $M$. tuberculosis $\mathrm{H} 37 \mathrm{Rv}$ has been described previously. ${ }^{56}$ Briefly, M. tuberculosis was grown to early-mid log phase in Middlebrook 7H9 media supplemented with 10\% OADC and 0.05\% Tween 80. Compounds were serially diluted 1:2 in 96 well microtiter plates. Bacteria were added to the plates and grown at $37^{\circ} \mathrm{C}$ for 6 days, Alamar blue was added and the plates were incubated for an additional $24 \mathrm{~h}$. The MIC was the drug concentration that inhibited visible growth and the reduction of Alamar blue in all the replicates.

In vivo studies-In vivo efficacy of TLM analogues was first investigated against a systemic infection model. Six-week old, pathogen free, male Swiss Webster mice weighing 27 to $30 \mathrm{~g}$ were used in this study. Animals were housed in the Division of Laboratory Animal Resources (DLAR) at Stony Brook University under BSL2 conditions and were provided ad libitum access to food and water through the entire experimental course. The animal study was approved by the Institutional Animal Care and Use Committee (IACUC) at Stony Brook University.

Methicillin resistant Staphylococcus aureus systemic infection model-The mice were rendered neutropenic by injecting cyclophosphamide 4 days and 1 day prior to MRSA infection. MRSA strain BAA 1762 was cultivated in Muller Hilton Broth to late exponential phase after which the bacteria were harvested and resuspended in Brain Heart Infusion (BHI) broth to give a concentration of $5 \times 10^{7}$ cells $/ \mathrm{mL}$. Systemic infection was initiated by injecting $0.5 \mathrm{~mL}$ of bacterial inoculum intraperitoneally. TLM analogues were dissolved in water/ethanol while vancomycin, as a positive control, was dissolved in water. Drug deliveries were given by subcutaneous injection $1 \mathrm{~h}$ and $25 \mathrm{~h}$ post infection with the dosage of $100 \mathrm{mg} / \mathrm{kg} / \mathrm{inj}$. The animals had access to food and water during the whole experiment. Survival was assessed every $12 \mathrm{~h}$ post infection until the end of the study. 
Klebsiella pneumoniae systemic infection model-A starting culture of $K$. pneumoniae KPC2ST258 was incubated in MH II broth to mid log phase $\left(\sim 10^{8} \mathrm{cfu} / \mathrm{mL}\right)$. Bacterial cells were harvested by centrifugation at 11,000 rpm for $3 \mathrm{~min}$. The inoculums were prepared by diluting cell pellets with freshly sterilized brain heart infusion (BHI) broth to $5 \times 10^{7} \mathrm{cfu} / \mathrm{mL}$. Systemic infection was initiated by administering $100 \mu \mathrm{L}$ of inoculum $\left(5 \times 10^{6} \mathrm{cfu}\right)$ via intraperitoneal (IP) injection. Infected animals received either TLManalogues or clinical antibiotics. All drugs were dissolved in the same vehicle which consisted of saline/PEG400/ethanol (40 / 20 / 40). Drugs were administered 1 h, 12 h and 24 h post infection via subcutaneous (SC) injection of $200 \mu \mathrm{L}$ containing $100 \mathrm{mg} / \mathrm{kg} / \mathrm{inj}$. Animals were monitored every $12 \mathrm{~h}$ following infection for 7 days. Dead animals were removed from the study immediately. Mice surviving at the end of the experiment were euthanized by $\mathrm{CO}_{2}$ inhalation as recommended by the American Veterinary Medical Association (AVMA). Survival curves were plotted based on the day that dead animals were discovered. Average survival time of each group was calculated.

Toxicity Studies-THP1, a human monocytic cell line cell line, was cultured in RPMI 1640 media supplemented with $10 \% \mathrm{FBS}, 2 \mathrm{mM}$ L-Glutamine, $1 \mathrm{mM}$ sodium pyruvate, penicillin (100 units $/ \mathrm{mL})$ and streptomycin $(100 \mu \mathrm{g} / \mathrm{mL})$ at $37{ }^{\circ} \mathrm{C}, 5 \% \mathrm{CO}_{2}$. The assay was performed in a 96 well plate format with a seeding density of $6.25 \times 10^{4}$ cells /well. The compounds were added at a starting concentration of $100 \mu \mathrm{g} / \mathrm{mL}$ and diluted $1: 2$ until 12.5 $\mu \mathrm{g} / \mathrm{mL}$. serially diluted 1:2 in 96 well microtiter plates. The cells were allowed to grow for 2 days. Alamar blue was added and the plates were incubated for an additional 24 hours. The $\mathrm{LD}_{50}$ was monitored based on reduction of Alamar blue in all the replicates.

\section{Supplementary Material}

Refer to Web version on PubMed Central for supplementary material.

\section{Acknowledgments}

Funding Sources: This work was funded by the National Institutes of Health grants GM102864 and AI044639 to PJT.

The authors would like to acknowledge Mr. Sajjad Hossain for help with cloning and Dr. Natasha M. Nesbitt for critical feedback on the manuscript.

\section{ABBREVIATIONS}

FASII Fatty acid biosynthesis type II pathway

KAS $\quad \beta$-Ketoacyl-ACP synthase

ACP acyl carrier protein

CoA Coenzyme A

TLM thiolactomycin

MIC minimum inhibitory concentration 
MSSA methicillin sensitive $S$. aureus

MRSA methicillin resistant $S$. aureus

\section{References}

1. Wright GD. The antibiotic resistome: the nexus of chemical and genetic diversity. Nat Rev Microbiol. 2007; 5:175-186. [PubMed: 17277795]

2. McGowan JE Jr. Resistance in nonfermenting gram-negative bacteria: multidrug resistance to the maximum. Am J Infect Control. 2006; 34:S29-37. discussion S64-73. [PubMed: 16813979]

3. Giamarellos-Bourboulis EJ, Antonopoulou A, Raftogiannis M, Koutoukas P, Tsaganos T, Tziortzioti V, Panagou C, Adamis T, Giamarellou H. Clarithromycin is an effective immunomodulator when administered late in experimental pyelonephritis by multidrug-resistant Pseudomonas aeruginosa. BMC Infect Dis. 2006; 6:31. [PubMed: 16504031]

4. CDC. Staphylococcus aureus resistant to vancomycin--United States, 2002. MMWR Morb Mortal Wkly Rep. 2002; 51:565-567. [PubMed: 12139181]

5. Magnuson K, Jackowski S, Rock CO, Cronan JE Jr. Regulation of fatty acid biosynthesis in Escherichia coli. Microbiol Rev. 1993; 57:522-542. [PubMed: 8246839]

6. Rock CO, Cronan JE. Escherichia coli as a model for the regulation of dissociable (type II) fatty acid biosynthesis. Biochim Biophys Acta. 1996; 1302:1-16. [PubMed: 8695652]

7. Campbell JW, Cronan JE Jr. Bacterial fatty acid biosynthesis: targets for antibacterial drug discovery. Annu Rev Microbiol. 2001; 55:305-332. [PubMed: 11544358]

8. Banerjee A, Dubnau E, Quemard A, Balasubramanian V, Um KS, Wilson T, Collins D, de Lisle G, Jacobs WR Jr. inhA, a gene encoding a target for isoniazid and ethionamide in Mycobacterium tuberculosis. Science. 1994; 263:227-230. [PubMed: 8284673]

9. Dessen A, Quemard A, Blanchard JS, Jacobs WR Jr, Sacchettini JC. Crystal structure and function of the isoniazid target of Mycobacterium tuberculosis. Science. 1995; 267:1638-1641. [PubMed: 7886450]

10. Quemard A, Sacchettini JC, Dessen A, Vilcheze C, Bittman R, Jacobs WR Jr, Blanchard JS. Enzymatic characterization of the target for isoniazid in Mycobacterium tuberculosis. Biochemistry. 1995; 34:8235-8241. [PubMed: 7599116]

11. Rawat R, Whitty A, Tonge PJ. The isoniazid-NAD adduct is a slow, tight-binding inhibitor of InhA, the Mycobacterium tuberculosis enoyl reductase: adduct affinity and drug resistance. Proc Natl Acad Sci U S A. 2003; 100:13881-13886. [PubMed: 14623976]

12. Escaich S, Prouvensier L, Saccomani M, Durant L, Oxoby M, Gerusz V, Moreau F, Vongsouthi V, Maher K, Morrissey I, Soulama-Mouze C. The MUT056399 inhibitor of FabI is a new antistaphylococcal compound. Antimicrob Agents Chemother. 2011; 55:4692-4697. [PubMed: 21825292]

13. Flamm RK, Rhomberg PR, Kaplan N, Jones RN, Farrell DJ. Activity of Debio1452, a Fabi inhibitor with potent activity against Staphylococcus aureus and coagulase-negative Staphylococcus spp., including multidrug-resistant strains. Antimicrob Agents Chemother. 2015; 59:2583-2587. [PubMed: 25691627]

14. Park HS, Yoon YM, Jung SJ, Yun IN, Kim CM, Kim JM, Kwak JH. CG400462, a new bacterial enoyl-acyl carrier protein reductase (FabI) inhibitor. Int J Antimicrob Agents. 2007; 30:446-451. [PubMed: 17723291]

15. Heath RJ, Rock CO. The Claisen condensation in biology. Nat Prod Rep. 2002; 19:581-596. [PubMed: 12430724]

16. Davies C, Heath RJ, White SW, Rock CO. The 1.8 A crystal structure and active-site architecture of $\beta$-ketoacyl-acyl carrier protein synthase III $(\mathrm{FabH})$ from Escherichia coli. Structure. 2000; 8:185-195. [PubMed: 10673437]

17. Jackowski S, Rock CO. Acetoacetyl-acyl carrier protein synthase, a potential regulator of fatty acid biosynthesis in bacteria. J Biol Chem. 1987; 262:7927-7931. [PubMed: 3294837]

18. Borgaro JG, Chang A, Machutta CA, Zhang X, Tonge PJ. Substrate recognition by $\beta$-ketoacyl-ACP synthases. Biochemistry. 2011; 50:10678-10686. [PubMed: 22017312] 
19. Yuan YQ, Sachdeva M, Leeds JA, Meredith TC. Fatty acid biosynthesis in Pseudomonas aeruginosa is initiated by the FabY class of $\beta$-ketoacyl acyl carrier protein synthases. J Bacteriol. 2012; 194:5171-5184. [PubMed: 22753059]

20. Wright HT, Reynolds KA. Antibacterial targets in fatty acid biosynthesis. Curr Opin Microbiol. 2007; 10:447-453. [PubMed: 17707686]

21. Young K, Jayasuriya H, Ondeyka JG, Herath K, Zhang C, Kodali S, Galgoci A, Painter R, BrownDriver V, Yamamoto R, Silver LL, Zheng Y, Ventura JI, Sigmund J, Ha S, Basilio A, Vicente F, Tormo JR, Pelaez F, Youngman P, Cully D, Barrett JF, Schmatz D, Singh SB, Wang J. Discovery of FabH/FabF inhibitors from natural products. Antimicrob Agents Chemother. 2006; 50:519-526. [PubMed: 16436705]

22. Ondeyka JG, Zink DL, Young K, Painter R, Kodali S, Galgoci A, Collado J, Tormo JR, Basilio A, Vicente F, Wang J, Singh SB. Discovery of bacterial fatty acid synthase inhibitors from a Phoma species as antimicrobial agents using a new antisense-based strategy. J Nat Prod. 2006; 69:377380. [PubMed: 16562839]

23. Wang J, Soisson SM, Young K, Shoop W, Kodali S, Galgoci A, Painter R, Parthasarathy G, Tang YS, Cummings R, Ha S, Dorso K, Motyl M, Jayasuriya H, Ondeyka J, Herath K, Zhang C, Hernandez L, Allocco J, Basilio A, Tormo JR, Genilloud O, Vicente F, Pelaez F, Colwell L, Lee SH, Michael B, Felcetto T, Gill C, Silver LL, Hermes JD, Bartizal K, Barrett J, Schmatz D, Becker JW, Cully D, Singh SB. Platensimycin is a selective FabF inhibitor with potent antibiotic properties. Nature. 2006; 441:358-361. [PubMed: 16710421]

24. Wang J, Kodali S, Lee SH, Galgoci A, Painter R, Dorso K, Racine F, Motyl M, Hernandez L, Tinney E, Colletti SL, Herath K, Cummings R, Salazar O, Gonzalez I, Basilio A, Vicente F, Genilloud O, Pelaez F, Jayasuriya H, Young K, Cully DF, Singh SB. Discovery of platencin, a dual FabF and FabH inhibitor with in vivo antibiotic properties. Proc Natl Acad Sci U S A. 2007; 104:7612-7916. [PubMed: 17456595]

25. Noto T, Miyakawa S, Oishi H, Endo H, Okazaki H. Thiolactomycin, a new antibiotic. III. In vitro antibacterial activity. J Antibiot (Tokyo). 1982; 35:401-410. [PubMed: 6980215]

26. Oishi H, Noto T, Sasaki H, Suzuki K, Hayashi T, Okazaki H, Ando K, Sawada M. Thiolactomycin, a new antibiotic. I. Taxonomy of the producing organism, fermentation and biological properties. J Antibiot (Tokyo). 1982; 35:391-395. [PubMed: 7096194]

27. Miyakawa S, Suzuki K, Noto T, Harada Y, Okazaki H. Thiolactomycin, a new antibiotic. IV. Biological properties and chemotherapeutic activity in mice. J Antibiot (Tokyo). 1982; 35:411419. [PubMed: 7096196]

28. Hamada S, Fujiwara T, Shimauchi H, Ogawa T, Nishihara T, Koga T, Nehashi T, Matsuno T. Antimicrobial activities of thiolactomycin against Gram-negative anaerobes associated with periodontal disease. Oral Microbiol Immunol. 1990; 5:340-345. [PubMed: 2098714]

29. Slayden RA, Lee RE, Armour JW, Cooper AM, Orme IM, Brennan PJ, Besra GS. Antimycobacterial action of thiolactomycin: an inhibitor of fatty acid and mycolic acid synthesis. Antimicrob Agents Chemother. 1996; 40:2813-2819. [PubMed: 9124847]

30. Price AC, Choi KH, Heath RJ, Li Z, White SW, Rock CO. Inhibition of $\beta$-ketoacyl-acyl carrier protein synthases by thiolactomycin and cerulenin. Structure and mechanism. J Biol Chem. 2001; 276:6551-6559. [PubMed: 11050088]

31. Kim P, Barry CE, Dowd CS. Novel route to 5-position vinyl derivatives of thiolactomycin: Olefination vs. deformylation. Tetrahedron Lett. 2006; 47:3447-3451. [PubMed: 16699591]

32. Machutta CA, Bommineni GR, Luckner SR, Kapilashrami K, Ruzsicska B, Simmerling C, Kisker $\mathrm{C}$, Tonge PJ. Slow onset inhibition of bacterial $\beta$-ketoacyl-acyl carrier protein synthases by thiolactomycin. J Biol Chem. 2010; 285:6161-6169. [PubMed: 20018879]

33. Kapilashrami K, Bommineni GR, Machutta CA, Kim P, Lai CT, Simmerling C, Picart F, Tonge PJ. Thiolactomycin-based $\beta$-ketoacyl-AcpM synthase A (KasA) inhibitors: fragment-based inhibitor discovery using transient one-dimensional nuclear overhauser effect NMR spectroscopy. J Biol Chem. 2013; 288:6045-6052. [PubMed: 23306195]

34. Senior SJ, Illarionov PA, Gurcha SS, Campbell IB, Schaeffer ML, Minnikin DE, Besra GS. Biphenyl-based analogues of thiolactomycin, active against Mycobacterium tuberculosis $\mathrm{mtFabH}$ fatty acid condensing enzyme. Bioorg Med Chem Lett. 2003; 13:3685-3688. [PubMed: 14552758] 
35. Senior SJ, Illarionov PA, Gurcha SS, Campbell IB, Schaeffer ML, Minnikin DE, Besra GS. Acetylene-based analogues of thiolactomycin, active against Mycobacterium tuberculosis $\mathrm{mtFabH}$ fatty acid condensing enzyme. Bioorg Med Chem Lett. 2004; 14:373-376. [PubMed: 14698162]

36. Bhowruth V, Brown AK, Senior SJ, Snaith JS, Besra GS. Synthesis and biological evaluation of a C5-biphenyl thiolactomycin library. Bioorg Med Chem Lett. 2007; 17:5643-5646. [PubMed: 17766110]

37. Jones SM, Urch JE, Kaiser M, Brun R, Harwood JL, Berry C, Gilbert IH. Analogues of thiolactomycin as potential antimalarial agents. J Med Chem. 2005; 48:5932-5941. [PubMed: 16161997]

38. Jones AL, Herbert D, Rutter AJ, Dancer JE, Harwood JL. Novel inhibitors of the condensing enzymes of the type II fatty acid synthase of pea (Pisum sativum). Biochem J. 2000; 347:205-209. [PubMed: 10727420]

39. Jones SM, Urch JE, Brun R, Harwood JL, Berry C, Gilbert IH. Analogues of thiolactomycin as potential anti-malarial and anti-trypanosomal agents. Bioorg Med Chem. 2004; 12:683-692. [PubMed: 14759729]

40. McFadden JM, Medghalchi SM, Thupari JN, Pinn ML, Vadlamudi A, Miller KI, Kuhajda FP, Townsend CA. Application of a flexible synthesis of (5R)-thiolactomycin to develop new inhibitors of type I fatty acid synthase. J Med Chem. 2005; 48:946-961. [PubMed: 15715465]

41. Kamal A, Shaik AA, Sinha R, Yadav JS, Arora SK. Antitubercular agents. Part 2: new thiolactomycin analogues active against Mycobacterium tuberculosis. Bioorg Med Chem Lett. 2005; 15:1927-1929. [PubMed: 15780635]

42. Still IWJ, Drewery MJ. Synthesis of 2-butenolide and tetronic acid analogs of thiolactomycin. J Org Chem. 1989; 54:290-295.

43. Kim P, Zhang YM, Shenoy G, Nguyen QA, Boshoff HI, Manjunatha UH, Goodwin MB, Lonsdale J, Price AC, Miller DJ, Duncan K, White SW, Rock CO, Barry CE, Dowd CS. Structure-activity relationships at the 5-position of thiolactomycin: An intact (5R)-isoprene unit is required for activity against the condensing enzymes from Mycobacterium tuberculosis and Escherichia coli. J Med Chem. 2006; 49:159-171. [PubMed: 16392800]

44. McFadden JM, Frehywot GL, Townsend CA. A flexible route to (5R)-thiolactomycin, a naturally occurring inhibitor of fatty acid synthesis. Org Lett. 2002; 4:3859-3862. [PubMed: 12599477]

45. Chambers MS, Thomas EJ, Williams DJ. An asymmetric-synthesis of thiotetronic acids using chirality transfer via an allyl xanthate-to-dithiocarbonate rearrangement - X-ray crystal-structure of (5r)-2,5-dihydro-4-hydroxy-5-methyl-3-phenyl-5-prop-1'-enyl-2-oxothiophene. J Chem Soc Chem Comm. 1987:1228-1230.

46. Chambers MS, Thomas EJ. Asymmetric synthesis of 5,5-disubstituted thiotetronic acids using an allyl xanthate to dithiocarbonate rearrangement: total synthesis of (5S)-thiolactomycin with revision of the absolute configuration of the natural. J Chem Soc, Perkin Trans 1. 1997:417-432.

47. Ohata K, Terashima S. Efficient synthesis of enantiomeric pairs of thiolactomycin and its 3demethyl derivative. Tetrahedron Lett. 2006; 47:2787-2791.

48. Ohata K, Terashima S. Synthesis and biological activity of enantiomeric pairs of 5vinylthiolactomycin congeners. Bioorg Med Chem Lett. 2007; 17:4070-4074. [PubMed: 17507223]

49. Ohata K, Terashima S. Synthesis and biological activity of enantiomeric pairs of 5-[(E)-cycloalk-2enylidenemethyl]thiolactomycin congeners. Bioorg Med Chem Lett. 2008; 18:5598-5600. [PubMed: 18805004]

50. Ohata K, Terashima S. Synthesis and biological activity of enantiomeric pairs of 5-(alk-2enyl)thiolactomycin and 5-[(e)-cycloalk-2-enylidenemethyl]thiolactomycin congeners. Chem Pharm Bull. 2009; 57:920-936. [PubMed: 19721252]

51. Dormann KL, Bruckner R. Variable synthesis of the optically active thiotetronic acid antibiotics thiolactomycin, thiotetromycin, and 834-B1. Angew Chem Int Ed Engl. 2007; 46:1160-1163. [PubMed: 17183501]

52. Schiebel J, Kapilashrami K, Fekete A, Bommineni GR, Schaefer CM, Mueller MJ, Tonge PJ, Kisker C. Structural basis for the recognition of mycolic acid precursors by KasA, a condensing 
enzyme and drug target from Mycobacterium tuberculosis. J Biol Chem. 2013; 288:34190-34204. [PubMed: 24108128]

53. Luckner SR, Machutta CA, Tonge PJ, Kisker C. Crystal structures of Mycobacterium tuberculosis KasA show mode of action within cell wall biosynthesis and its inhibition by thiolactomycin. Structure. 2009; 17:1004-1013. [PubMed: 19604480]

54. Hori K, Arai M, Nomura K, Yoshii E. An efficient 3(C)-acylation of tetramic acids involving acyl migration of 4(O)-acylates. Chem Pharm Bull. 1987; 35:4368-4371.

55. Jeong YC, Moloney MG. Synthesis of and tautomerism in 3-acyltetramic acids. J Org Chem. 2011; 76:1342-1354. [PubMed: 21250713]

56. am Ende CW, Knudson SE, Liu N, Childs J, Sullivan TJ, Boyne M, Xu H, Gegina Y, Knudson DL, Johnson F, Peloquin CA, Slayden RA, Tonge PJ. Synthesis and in vitro antimycobacterial activity of B-ring modified diaryl ether InhA inhibitors. Bioorg Med Chem Lett. 2008; 18:3029-3033.

[PubMed: 18457948] 


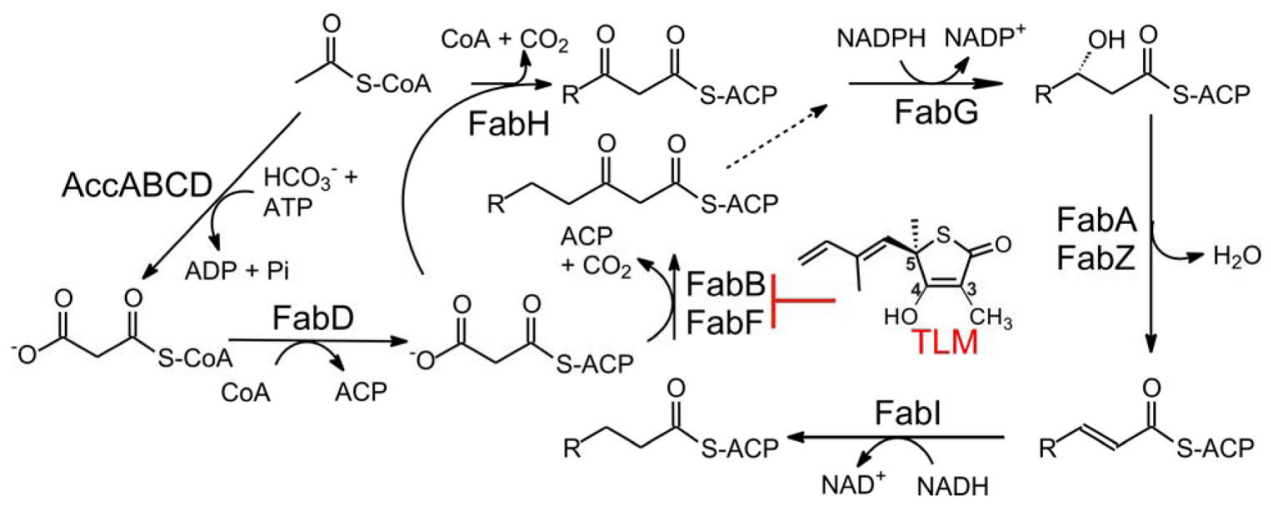

Figure 1.

Fatty Acid Biosynthesis pathway in E. coli. $\beta$-Ketoacyl-ACP synthases (FabB, FabF and $\mathrm{FabH}$ ) catalyze the decarboxylative Claisen condensation of malonyl-ACP with the acyl primer. While FabB and FabF are responsible for the elongation step of FASII, FabH initiates FASII by synthesizing acetoacetyl-ACP. Thiolactomycin (TLM), a natural product thiolactone, preferentially inhibits the FabB and FabF $\beta$-ketoacyl-ACP synthases ${ }^{15}$. 
A. Methicillin resistant S. aureus BAA1762

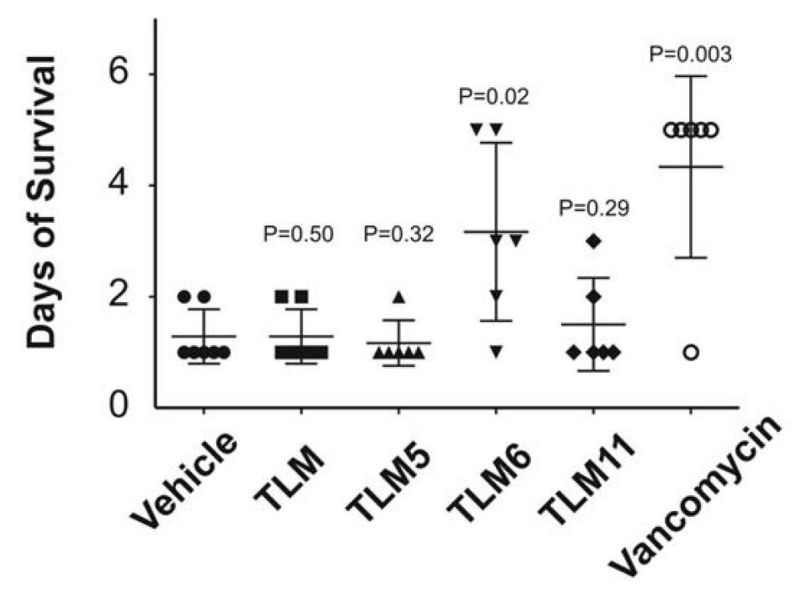

B. $\quad$ K. pneumoniae KPC2ST258

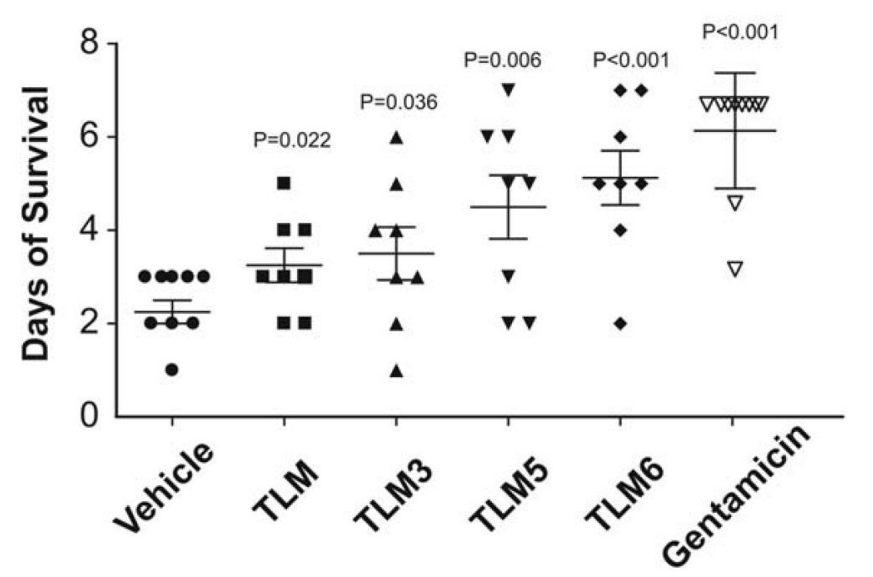

Figure 2.

Average survival time of mice infected with, A) Methicillin resistant $S$. aureus BAA1762 and treated with vehicle $(\boldsymbol{\bullet}, \mathrm{n}=7)$, TLM $(\boldsymbol{\bullet}, \mathrm{n}=7)$, TLM $5(\boldsymbol{\Delta}, \mathrm{n}=6)$, TLM $6(\boldsymbol{\nabla}, \mathrm{n}=6)$, TLM $11(\diamond, \mathrm{n}=6)$ and vancomycin $(\bigcirc, \mathrm{n}=6) ; \mathrm{B})$ K. pneumoniae KPC2ST258 and treated with vehicle $(\boldsymbol{\bullet}, \mathrm{n}=10), \operatorname{TLM}(\boldsymbol{\square}, \mathrm{n}=8)$, TLM $3(\boldsymbol{\Delta}, \mathrm{n}=8)$, TLM $5(\boldsymbol{\nabla}, \mathrm{n}=8)$, TLM $6(\bullet, \mathrm{n}=8)$ and Gentamicin $(\nabla, \mathrm{n}=10)$. The infected animals were monitored twice a day for 5 days post infection for the MRSA infection model and 7 days for the $K$. pneumoniae infection model. The average survival time was calculated at the end of each study. The one-tailed $t$ test was used to calculate the $\mathrm{p}$ value of each data set with respect to the vehicle control. 


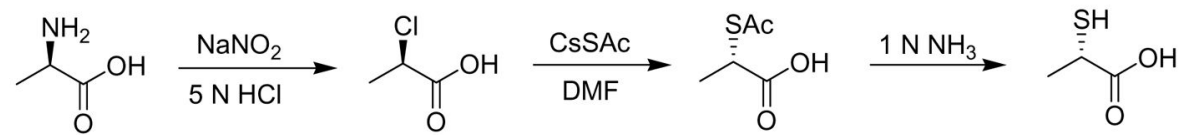

D-Alanine
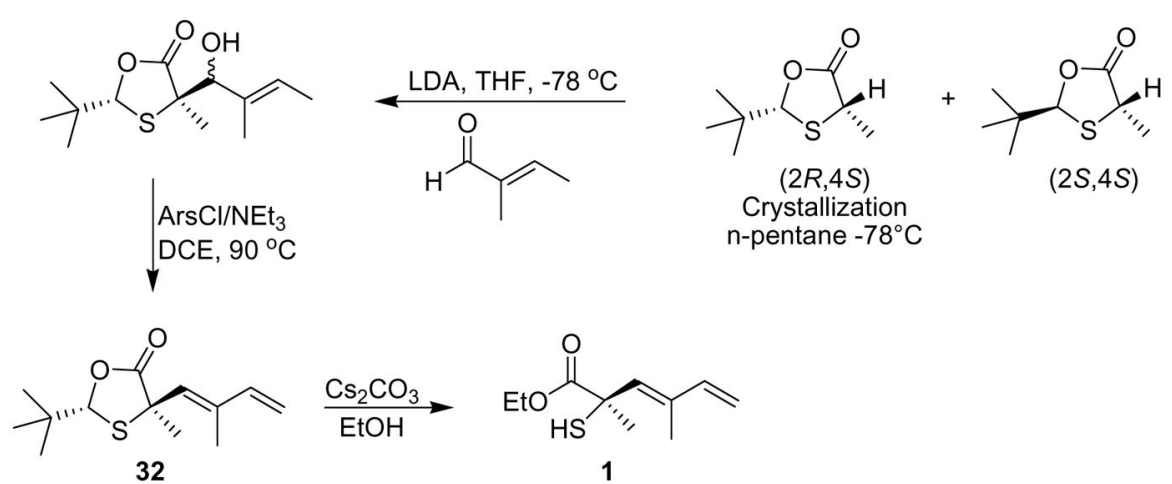

Scheme 1.

Synthesis of building blocks 32 and 1. 


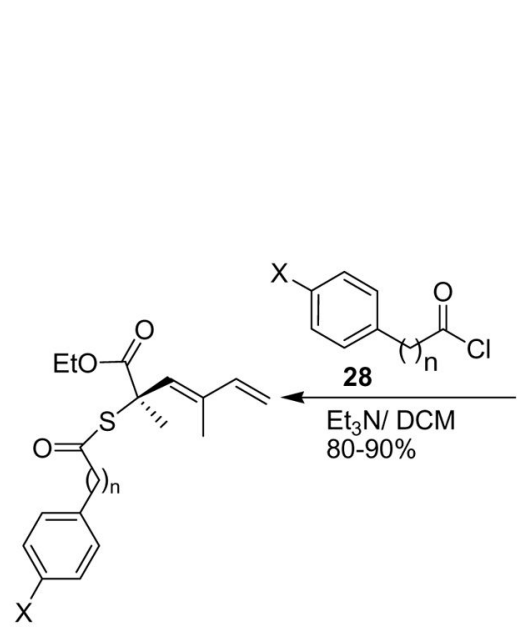

12a: $n=3, x=C$

13a: $n=3, X=\mathrm{CH}_{3}$

14a: $n=5, X=F$

15a: $n=5, X=C l$

16a: $n=5, X=$ Phenyl

19a: $n=5, X=B r$

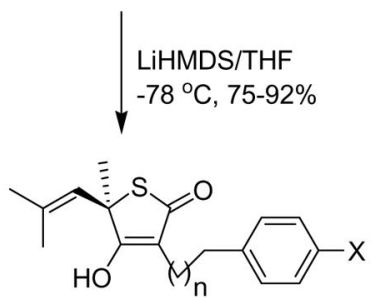

TLM 12: $\mathrm{n}=1, \mathrm{X}=\mathrm{Cl}$

TLM 13: $\mathrm{n}=1, \mathrm{X}=\mathrm{CH}_{3}$

TLM 14: $\mathrm{n}=3, \mathrm{X}=\mathrm{F}$

TLM 15: $\mathrm{n}=3, \mathrm{X}=\mathrm{Cl}$

TLM 16: $n=3, X=$ Phenyl

TLM 19: $n=3, X=B r$

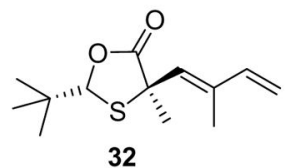

32

$\mathrm{Cs}_{2} \mathrm{CO}_{3}$

$\mathrm{EtOH}$

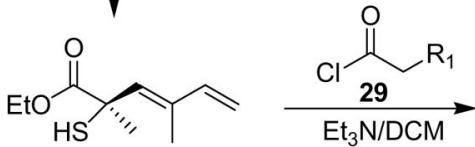

1

1a: $\mathrm{R}_{1}=\mathrm{CH}_{3}, 86 \%$

2a: $R_{1}=\mathrm{H}, 84 \%$

3a: $\mathrm{R}_{1}=\mathrm{CH}_{2} \mathrm{CH}_{3} 82 \%$

4a: $\mathrm{R}_{1}=\mathrm{CH}_{2} \mathrm{CH}_{2} \mathrm{CH}_{3} 84 \%$
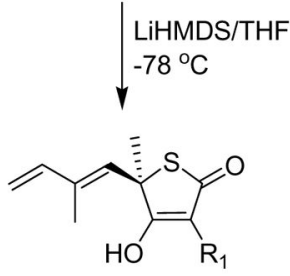

TLM: $\mathrm{R}_{1}=\mathrm{CH}_{3}, 80 \%$

TLM 2: $\mathrm{R}_{1}=\mathrm{H}, 58 \%$

TLM 3: $\mathrm{R}_{1}=\mathrm{CH}_{2} \mathrm{CH}_{3}, 75 \%$

TLM 4: $\mathrm{R}_{1}=\mathrm{CH}_{2} \mathrm{CH}_{2} \mathrm{CH}_{3}, 78 \%$

Scheme 2.

Synthesis of C3 analogs of $5 R-(+)$-Thiolactomycin (TLM $2-4,12-16,19$ ) 
<smiles>C=C/C(C)=C/[C@@]1(C)SC(=O)C=C1O</smiles><smiles>[CH]1[CH]C=C1</smiles><smiles>CC(C)(C)OC(=O)CBr</smiles><smiles>C=C/C(C)=C/[C@@]1(C)SC(=O)C=C1OCC(=O)O</smiles>

TLM 2

ii. $25 \%$ TFA/DCM, $24 \mathrm{~h}$<smiles>[R]CCNC(C)CC(C)C(C)=O</smiles><smiles>C=C/C(C)=C/[C@@]1(C)SC(=O)C=C1O</smiles>

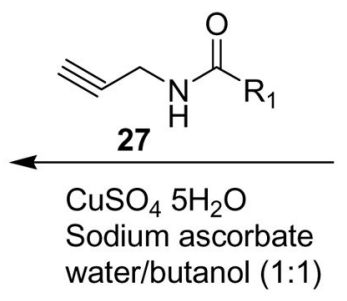<smiles>[R]CCNC(=O)COC1=CC(=O)S[C@@]1(C)/C=C(\C)C=C</smiles>

TLM 22: $\mathrm{R}=\mathrm{N}_{3}$ TLM 23: $R=$ NHBoc<smiles></smiles>

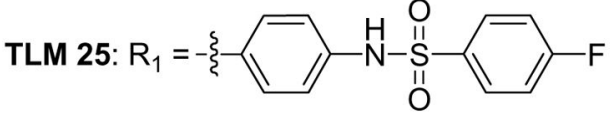

Scheme 3.

Synthesis of C4 analogs of $5 R$-(+)-Thiolactomycin (TLM $22-25$ ) 


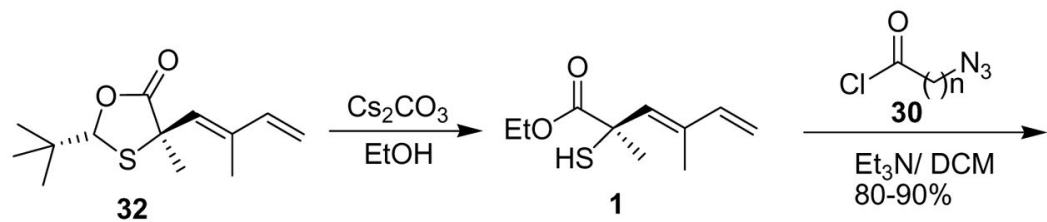<smiles>C=C/C(C)=C/[C@@](C)(SC(=O)C=N)C(=O)OCC</smiles>

18a: $\mathrm{n}=5,86 \%$

20a: $n=4,84 \%$

iHMDS/THF $-78^{\circ} \mathrm{C}, 75 \%$
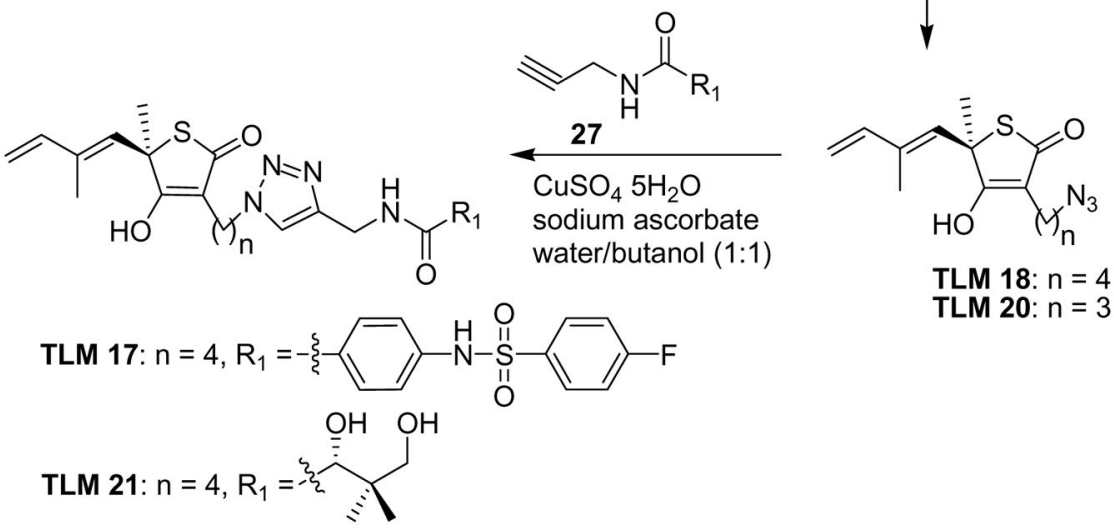

TLM 18: $\mathrm{n}=4$ TLM 20: $n=3$

Scheme 4.

Synthesis of C3 analogs of $5 R$-(+)-Thiolactomycin (TLM 17, 18, 20, 21) 

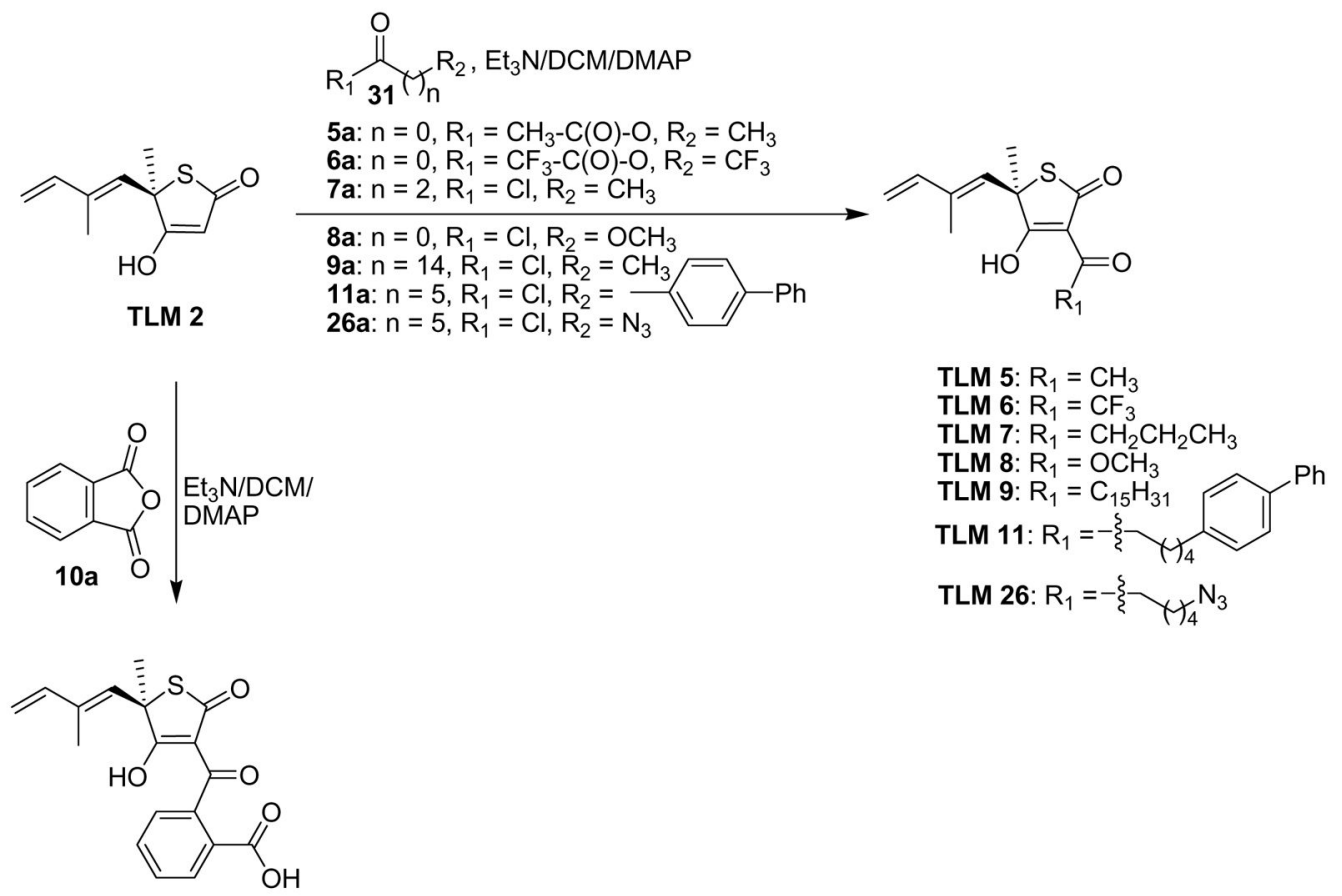

TLM 10

Scheme 5 .

Synthesis of C3 analogs of $5 R$-(+)-Thiolactomycin (TLM 5-11, 26) 


\section{Table 1}

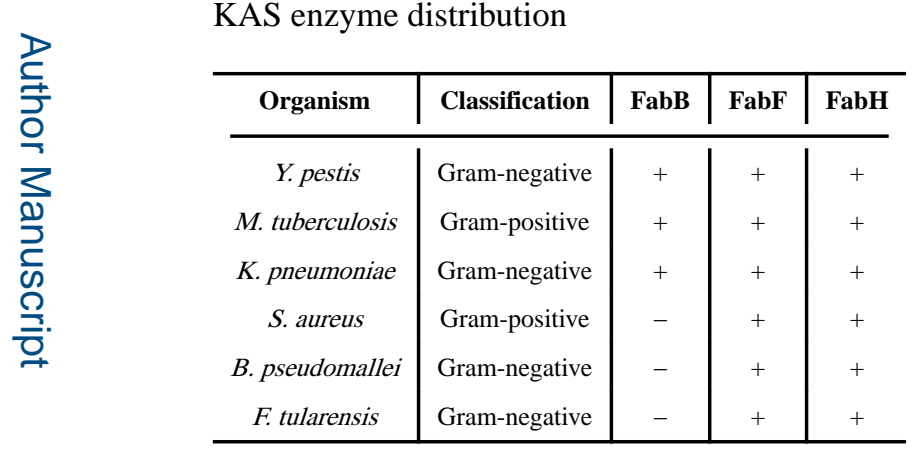

The $\beta$-Ketoacyl-ACP synthases present in the bacteria used in the study 

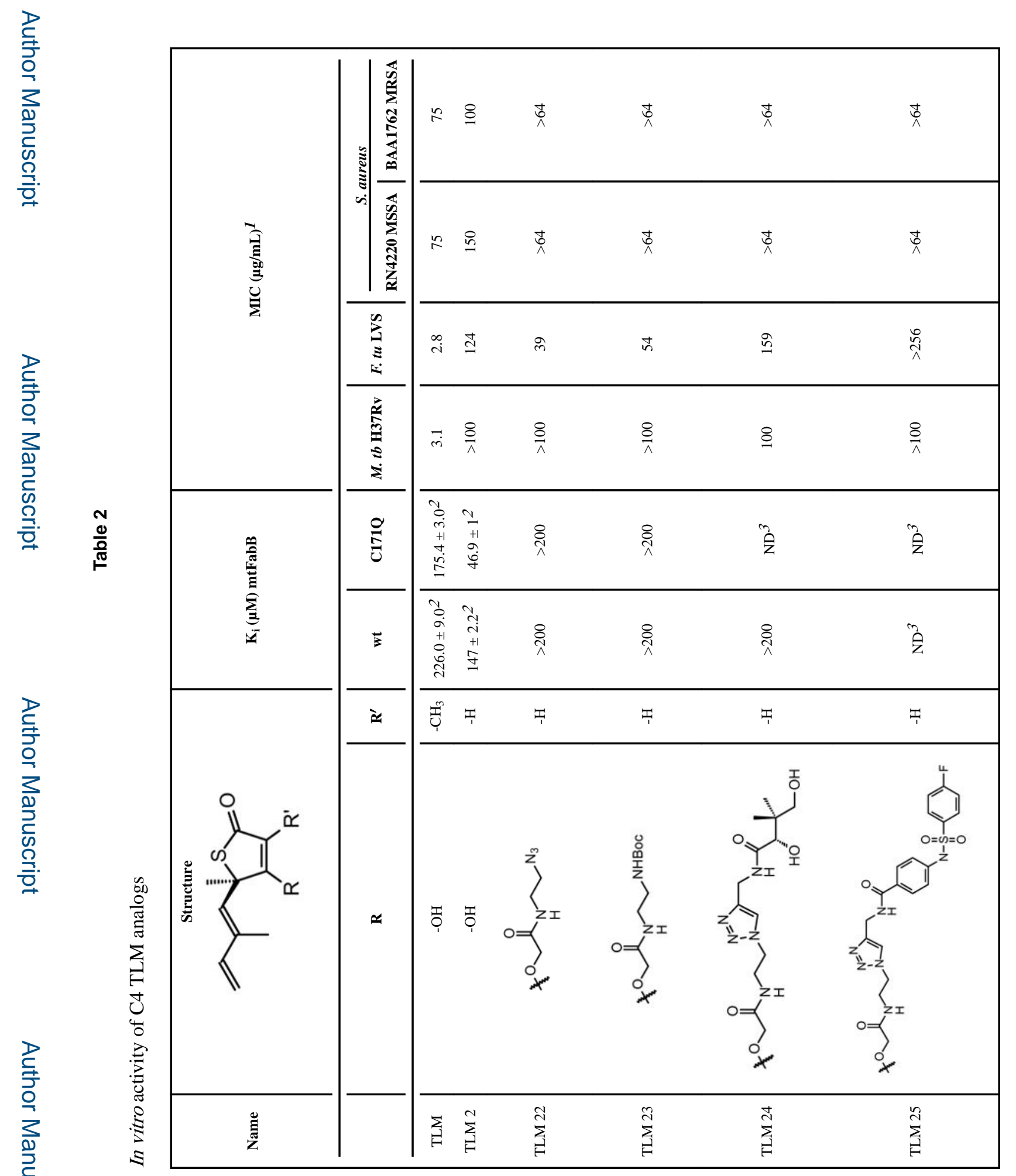


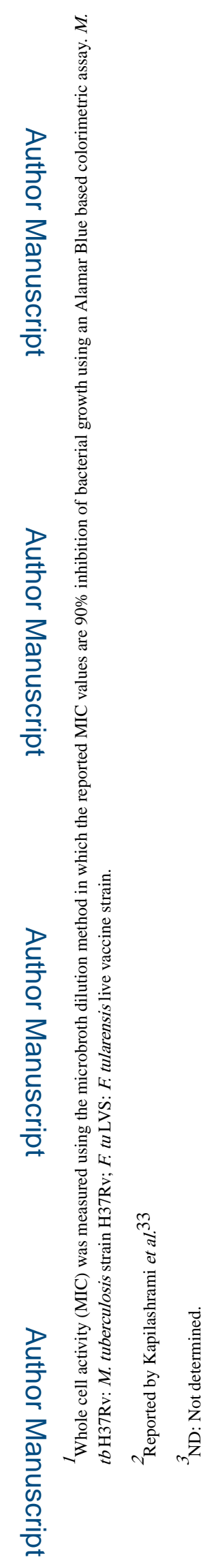



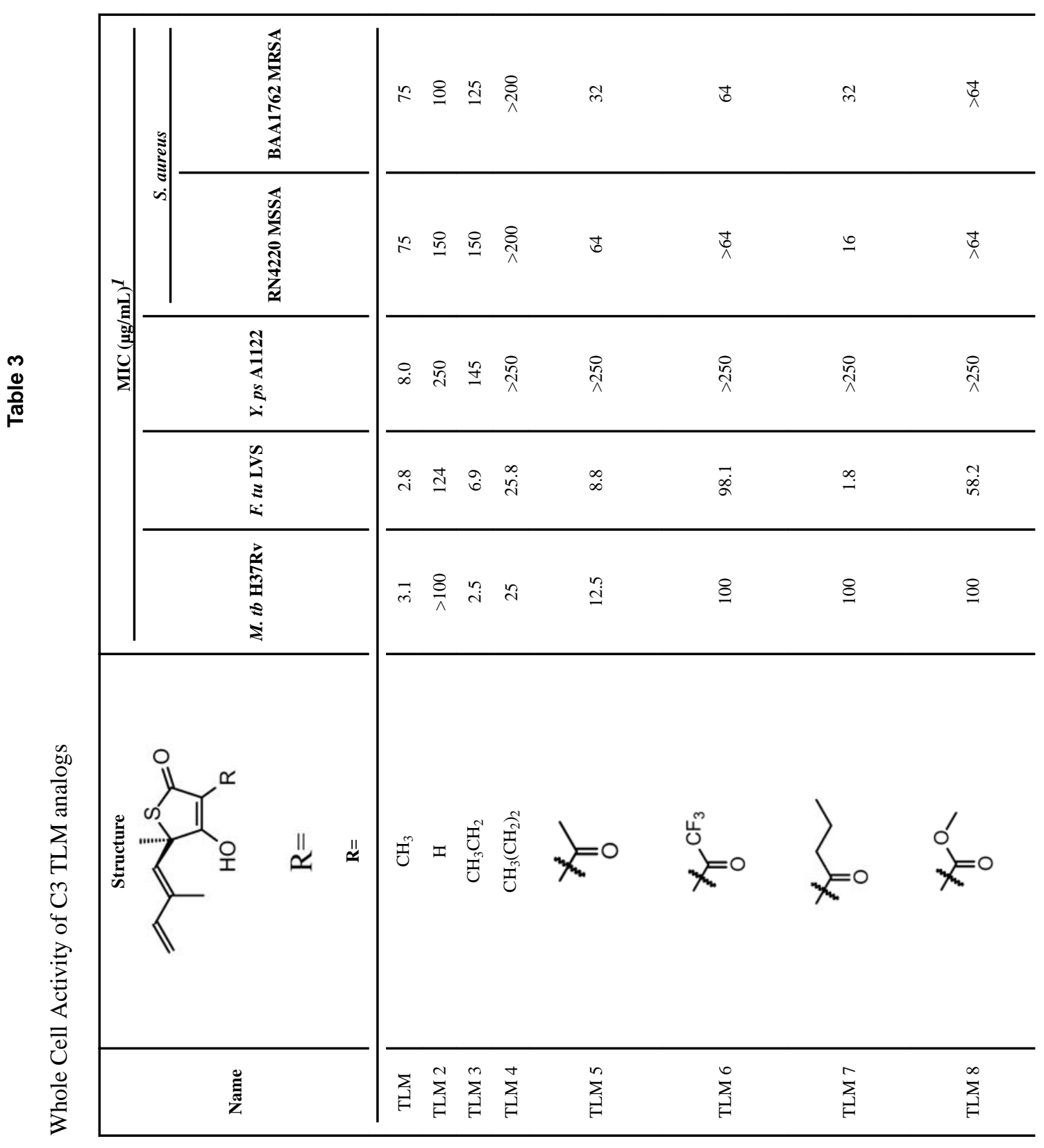

JMed Chem. Author manuscript; available in PMC 2017 June 09. 


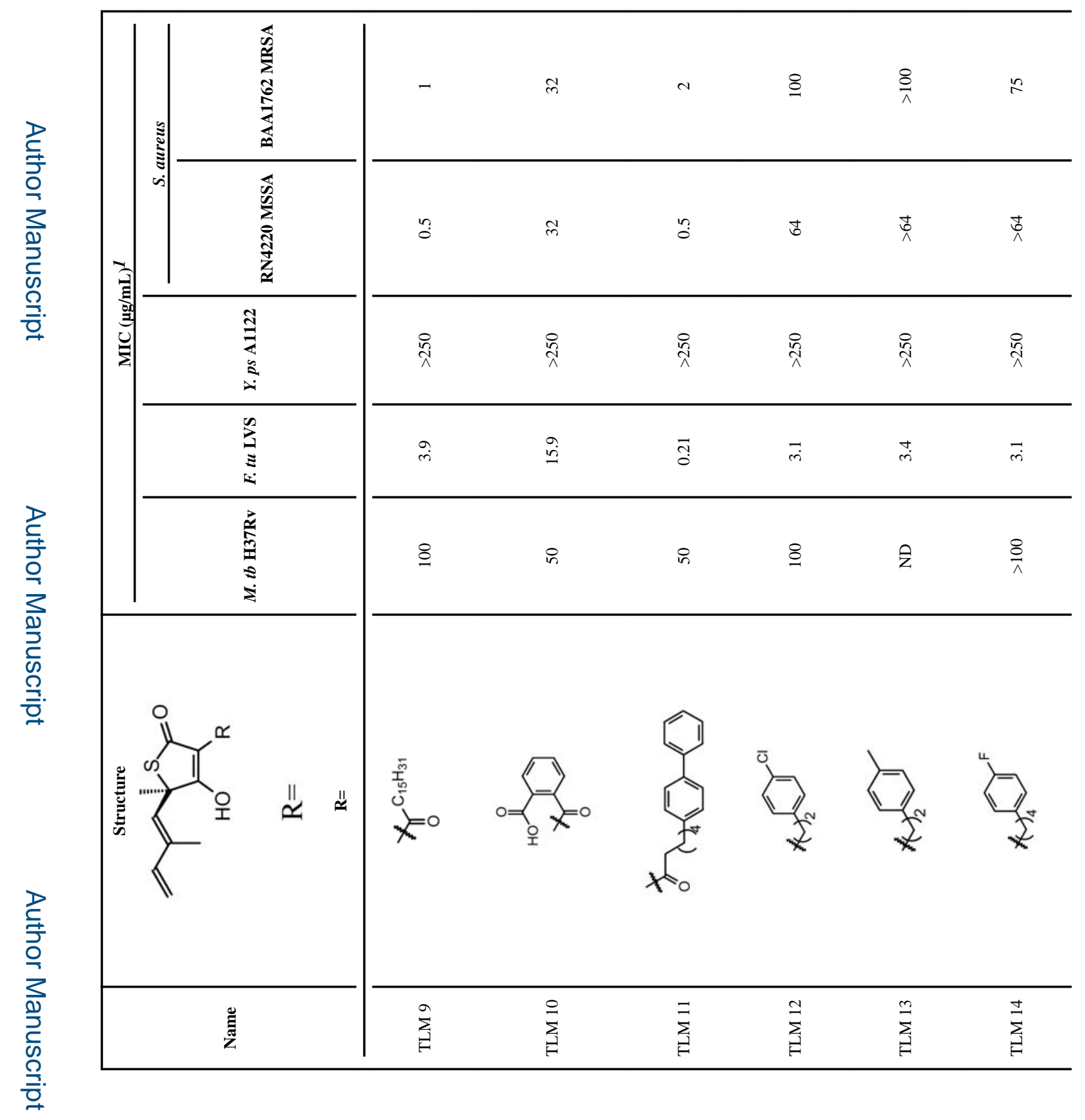

로을 


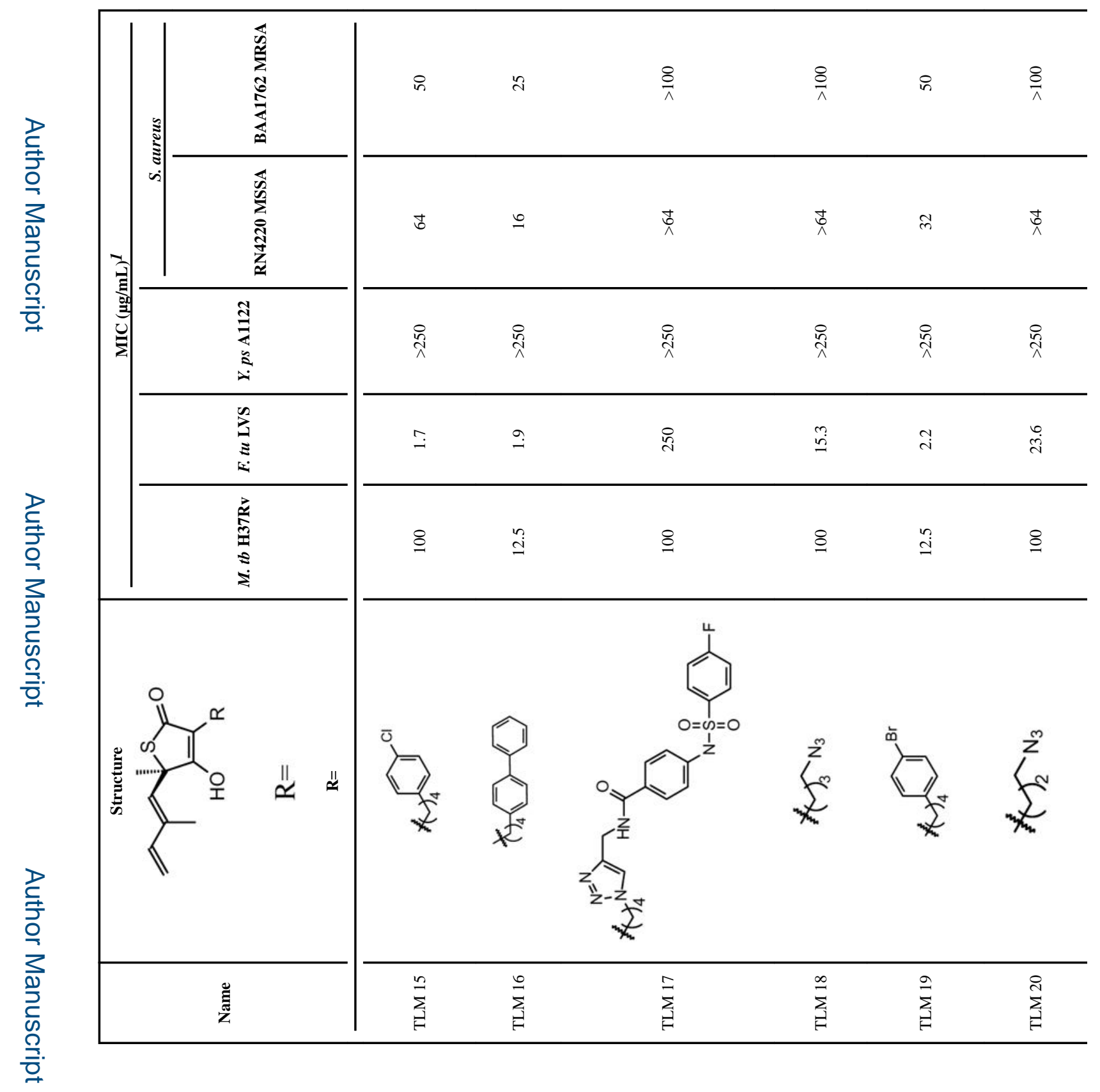

로을 


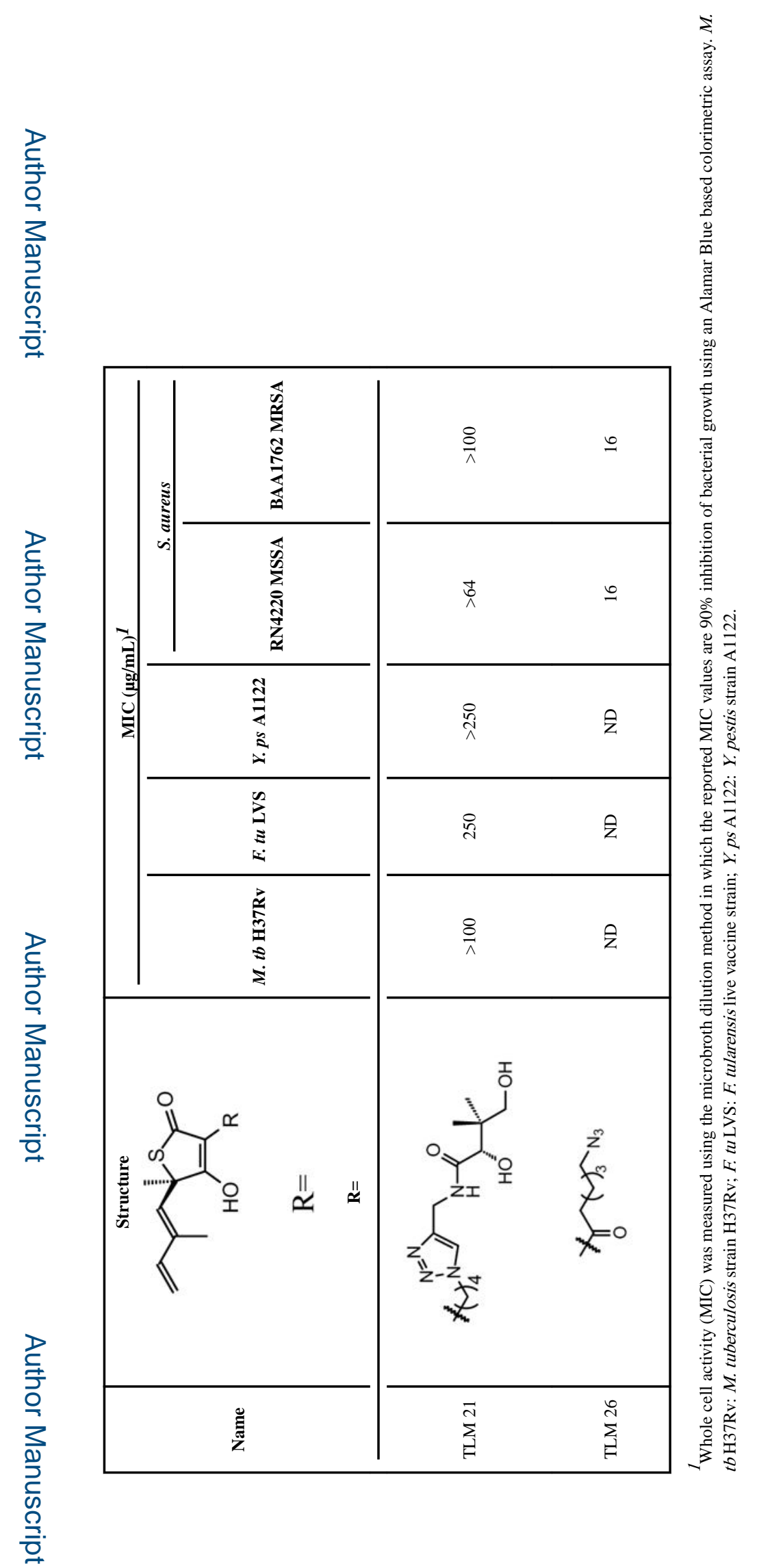

JMed Chem. Author manuscript; available in PMC 2017 June 09. 


\section{Table 4}

Inhibition of saFabF by TLM analogs

\begin{tabular}{c|c|c}
\hline Inhibitors & wt saFabF $\mathbf{K}_{\mathbf{d}}(\boldsymbol{\mu M})$ & $\mathbf{C 1 6 5 Q} \mathbf{s a F a b F} \mathbf{K}_{\mathbf{d}}(\boldsymbol{\mu M})$ \\
TLM & $107.0 \pm 3.0$ & $88.0 \pm 2.0$ \\
TLM 5 & $14.2 \pm 0.8$ & $11.9 \pm 0.6$ \\
TLM 6 & $58.4 \pm 0.7$ & $56.2 \pm 0.7$ \\
TLM 9 & $>200$ & $\mathrm{ND}$ \\
TLM 11 & $35.2 \pm 1.0$ & $33.0 \pm 0.7$ \\
TLM 16 & $131.0 \pm 1.0$ & $\mathrm{ND}$ \\
TLM 18 & $107.2 \pm 1.4$ & $\mathrm{ND}$ \\
TLM 19 & $92 \pm 1.0$ & $\mathrm{ND}$ \\
\hline
\end{tabular}

Dissociation constants were determined using the fluorescence based assay described previously ${ }^{32}$. The inhibitor stock solutions, dissolved in DMSO or $50 \mathrm{mM}$ Tris- $\mathrm{HCl}, 150 \mathrm{mM} \mathrm{NaCl} \mathrm{pH} 8.5$, were titrated into the enzyme $(1 \mu \mathrm{M})$ in $50 \mathrm{mM}$ Tris- $\mathrm{HCl}, 150 \mathrm{mM} \mathrm{NaCl}, 1 \mathrm{mM} \mathrm{DTT}$, pH 8.5 . Changes in fluorescence were monitored using excitation and emission wavelengths of $280 \mathrm{~nm}$ and $337 \mathrm{~nm}$, respectively. By convention, $K_{d}$ is used to identify the dissociation constant for wild-type KAS enzymes, while $\mathrm{K}_{\mathrm{i}}$ and $\mathrm{K}_{\mathrm{i}}^{*}$ are used for the initial and final equilibrium constants for inhibitors binding to acyl KAS enzyme mimic. ND: Not determined. 